\title{
FLOER COHOMOLOGY AND DISC INSTANTONS OF LAGRANGIAN TORUS FIBERS IN FANO TORIC MANIFOLDS*
}

\author{
CHEOL-HYUN $\mathrm{CHO}^{\dagger}$ AND YONG-GEUN $\mathrm{OH}^{\ddagger}$
}

\begin{abstract}
In this paper, we first provide an explicit description of all holomorphic discs ("disc instantons") attached to Lagrangian torus fibers of arbitrary compact toric manifolds, and prove their Fredholm regularity. Using this, we compute Fukaya-Oh-Ohta-Ono's (FOOO's) obstruction (co)chains and the Floer cohomology of Lagrangian torus fibers of Fano toric manifolds. In particular specializing to the formal parameter $T^{2 \pi}=e^{-1}$, our computation verifies the folklore that FOOO's obstruction (co)chains correspond to the Landau-Ginzburg superpotentials under the mirror symmetry correspondence, and also proves the prediction made by K. Hori about the Floer cohomology of Lagrangian torus fibers of Fano toric manifolds. The latter states that the Floer cohomology (for the parameter value $T^{2 \pi}=e^{-1}$ ) of all the fibers vanish except at a finite number, the Euler characteristic of the toric manifold, of base points in the momentum polytope that are critical points of the superpotential of the Landau-Ginzburg mirror to the toric manifold. In the latter cases, we also prove that the Floer cohomology of the corresponding fiber is isomorphic to its singular cohomology.

We also introduce a restricted version of the Floer cohomology of Lagrangian submanifolds, which is a priori more flexible to define in general, and which we call the adapted Floer cohomology. We then prove that the adapted Floer cohomology of any non-singular torus fiber of Fano toric manifolds is well-defined, invariant under the Hamiltonian isotopy, which is isomorphic to the Bott-Morse Floer cohomology of the fiber.
\end{abstract}

Key words. Floer cohomology, Lagrangian submanifold, holomorphic disc, toric manifold, Landau-Ginzburg model.

AMS subject classifications. 53D12, 53D40, 14J45, 14J32.

1. Introduction. Floer cohomology of Lagrangian intersections was introduced by Floer $[\mathrm{Fl}]$ in symplectic geometry. Since then, its construction has been further generalized [O1] and an obstruction theory to its definition has been developed by Fukaya-Oh-Ohta-Ono [FOOO]. It has been proven to be a powerful tool in studying various problems in symplectic geometry (see [Fl], [O4], [Che], [P], [Se], [FOOO], [BC], and $[\mathrm{TY}]$, for example). The theory itself was greatly enhanced by the advent of the Fukaya category [Fu1] and the homological mirror symmetry proposal by Kontsevich [Ko], and also by the open string theory of $D$-branes in many physics papers, among which $[\mathrm{HV}],[\mathrm{H}]$ will be the most relevant to the content of the present paper.

Even in the midst of these theoretical enhancement and successful applications of the Floer theory, actual computation of Floer cohomology itself for specific examples remains to be a non-trivial task, especially with $\mathbb{Z}$-coefficients (not just with $\mathbb{Z}_{2}$-coefficients), except for the cases where there is no quantum contribution [Fl] or for the case of real manifolds i.e., the fixed point sets of anti-holomorphic involutions [O2], [FOOO]. Indeed, computation of the Floer cohomology in the presence of nontrivial holomorphic discs requires detailed understanding of the quantum contribution of the holomorphic discs (or the effect of "open string instantons" in the physics terminology) to the cohomology of the Lagrangian submanifolds. In this respect, the recent

\footnotetext{
*Received September 2, 2005; accepted for publication May 18, 2006.

†Department of Mathematics, Northwestern University, Evanston, IL 60208, USA (cho@math. northwestern.edu); and Seoul National University, Seoul, Korea.

${ }_{\ddagger}^{\ddagger}$ Department of Mathematics, University of Wisconsin, Madison, WI 53706, USA (oh@math. wisc.edu); and Korea Institute for Advanced Study, 207-43 Cheongryangri-dong Dongdaemun-gu, Seoul 130-012, Korea. The senior author is partially supported by the NSF grant \#DMS 0203593, a grant of the 2000 Korean Young Scientist Prize, and the Vilas Research Award of University of Wisconsin.
} 
computation [Cho1] by the junior author of the Floer cohomology of the Clifford torus in $\mathbb{P}^{n}$ sheds some light on a general procedure of computing the Floer cohomology "by direct calculation of disc instanton effects" in the context of $A$-model without relying on the $B$-model calculations and the mirror symmetry correspondence, which still remains conjectural.

In this paper, we extend this computation and compute the (adapted) Floer cohomology of all the non-singular torus fibers of smooth Fano toric varieties equipped with symplectic (Kähler) form subject to a convex restriction. As in [Cho1], we will carry this out by computing the Bott-Morse version $H F^{B M}\left(L ; J_{0}\right)$ of the Floer cohomology of Lagrangian submanifold $L$ that was introduced in [FOOO]. Our computation, when the Floer cohomology is twisted with the flat line bundles and the formal parameter $T$ is set $T^{2 \pi}=e^{-1}$, verifies the prediction made by Hori-Vafa [HV] for the Lagrangian torus fibers of Fano toric manifolds based on the mirror symmetry correspondence via the linear sigma models [Wi], [HV]. However due to a technical problem regarding rectifiability of the moduli chain with a sphere bubble component, we impose rather a strict assumption 6.1, convexity of the symplectic manifold, which is not used in any other parts of the paper. In this case, all the relevant moduli chain is already rectifiable without perturbing the standard complex structure of the toric manifold if we equip the latter with the canonical symplectic form which naturally arises from the linear sigma model description of toric manifolds of Witten [Wi]. But we believe, based on Theorem 6.1 and Corollary 6.5, the computation should go through in general toric Fano manifolds, which we leave for a further research.

In the point of view of the obstruction theory developed in [O1], [FOOO], a priori, the torus fibers of general toric manifolds are neither monotone nor unobstructed, and may carry holomorphic discs of non-positive Maslov indices. Recall that the Clifford torus is also obstructed as an object in the $A_{\infty}$-category [O1], [FOOO], but the fact that it is monotone enables one to define the Floer cohomology [O1],[OH] which the junior author computed in [Cho1]. Combination of these facts prevent us from directly applying the general construction of the Floer cohomology from [FOOO] and forces us to manually construct a restricted version of the Floer cohomology and to prove the invariance property. For this purpose, some specific geometry of the moduli of holomorphic discs associated to the pair $\left(L, J_{0}\right)$ of the torus fiber $L$ and the canonical complex structure $J_{0}$ on the toric variety will play an essential role both for the definition and computation of the Floer cohomology. We will prove that there exists no non-constant holomorphic discs of non-positive Maslov indices for the torus fibers, although its Hamiltonian deformations of them may allow such (pseudo-)holomorphic discs. Our definition of the adapted Floer cohomology exploits this specific feature of the pair $\left(L, J_{0}\right)$. We call this version of the Floer cohomology the adapted Floer cohomology. It appears that in general this adapted Floer cohomology is more flexible to define and exploits best specific features of the moduli of holomorphic discs of the given pair $\left(L, J_{0}\right)$. In our particular situation of convex toric manifolds, this adapted Floer cohomology is known to be isomorphic to the Bott-Morse Floer cohomology $H F^{B M}\left(L ; J_{0}\right)$ with the bounding chain $b=0$ in the sense of [FOOO]. We refer to the final version of [FOOO] for more detailed explanation on this aspect.

In this paper, we compute $H F^{B M}\left(L ; J_{0}\right)$ with the bounding chain $b=0$ and its computation largely follows the scheme used by the first named author [Cho1]: Firstly, we derive general Maslov index formula of holomorphic discs in terms of the intersection number of natural divisors associated to the toric manifolds. Secondly we explicitly classify all the holomorphic discs and prove the Fredholm regularity of 
the discs. Then using this information, we compute $H F^{B M}\left(L ; J_{0}\right)$ with respect to the complex structure $J_{0}$.

In the course of our computation, we also derive an area formula Theorem 8.1 for the holomorphic discs of the Maslov index 2 (and so of all holomorphic discs) in terms of the location of the base point of the Lagrangian fiber and the relative homology class of the disc (or the divisor of the toric manifold that the disc intersect). This formula is crucial for our proof of the prediction that the base points in the momentum polytope at which the corresponding fiber has non-trivial Floer cohomology are indeed those corresponding to the critical points of the superpotential of the Landau-Ginzburg mirror.

We would like to emphasize that the mirror symmetry prediction made both in the Kontsevich proposal or by physicists does not really concern the standard Floer cohomology in symplectic geometry which uses the Novikov ring as its coefficients, but its convergent power series version. One byproduct of our classification of disc instantons is that this latter version of the Floer cohomology is defined and so substitution of the formal parameter $T^{2 \pi}$ by the number $e^{-1}$ is allowed in the Fano toric case. However the latter version of the Floer cohomology is not known to be invariant in general under the Hamiltonian isotopy of the Lagrangian torus fiber and so the mirror symmetry prediction concerns the Kähler geometry of the Lagrangian torus fibers (with respect to the natural complex structure $J_{0}$ and the Kähler form $\omega$ ), rather than the symplectic geometry of its Hamiltonian isotopy class. For example, it is possible that a fiber has trivial Floer cohomology with Novikov ring as its coefficients, but non-trivial one with the parameter value $T^{2 \pi}=e^{-1}$ (see section 13 for an explicit example of Hirzebruch surfaces).

Our work also provides some concrete mathematical evidence in the toric case for the conjectural relation between the superpotential and the "open Gromov-Witten invariants" which has been advocated by physicists (see [KKLM] for example). More precisely, we verify that under the mirror symmetry correspondence of a torus fiber, the one-point open Gromov-Witten invariant, which is essentially FOOO's obstruction chain [FOOO], maps to the superpotential $W$ of the Landau-Ginzburg mirror, and two-point invariants, which is essentially the Floer differential $\delta_{2}\langle p t\rangle$ in the Bott-Morse setting, maps to the derivative $\frac{\partial W}{\partial \Theta}$. We refer to section 15 for more discussion on this point or [Cho2] for a generalization of this correspondence.

One general distinction between the Fano and the non-Fano cases lies in the transversality property of the singular strata of various compactified moduli spaces. More precisely, non-Fano manifolds carry spheres of non-positive Chern numbers and so the compactified moduli space may contain singular strata that contain sphere bubbles (especially their multiple covers) of non-positive Chern numbers. As the study in [FOOO] demonstrated, such problems in the moduli space of holomorphic discs in relation to the Floer theory (or to open Gromov-Witten invariants) are much more troublesome than the case of spheres. We refer to section 16 for more detailed discussion on this.

We like to thank K. Hori for explaining us the mirror symmetry correspondence via the Landau-Ginzburg models and his $B$-model calculation that leads to his conjectural description of the Floer cohomology of the fibers of Fano toric manifolds. The junior author would like to thank L. Borisov and S. Hu for helpful discussions. Both authors thank K. Fukaya, H. Ohta and K. Ono for their interest in the results of this paper and some interesting discussion on the homotopy identity defined in [FOOO] during the senior author's visit of Kyoto University in August, 2003. 
2. Complex structures on toric manifolds. We consider smooth and compact toric varieties. Here we closely follow the Batyrev [B1] with minor notational changes (See M. Audin [Au] for more details)

In order to obtain an n-dimensional compact toric manifold $V$, we need a combinatorial object $\Sigma$, a complete fan of regular cones, in a $n$-dimensional vector space over $\mathbb{R}$.

Let $N$ be the lattice $\mathbb{Z}^{n}$, and let $M=\operatorname{Hom}_{\mathbb{Z}}(N, \mathbb{Z})$ be the dual lattices of rank $N$. Let $N_{\mathbb{R}}=N \otimes \mathbb{R}$ and $M_{\mathbb{R}}=M \otimes \mathbb{R}$.

Definition 2.1. A convex subset $\sigma \subset N_{\mathbb{R}}$ is called a regular $k$-dimensional cone $(k \geq 1)$ if there exists $k$ linearly independent elements $v_{1}, \cdots, v_{k} \in N$ such that

$$
\sigma=\left\{a_{1} v_{1}+\cdots+a_{k} v_{k} \mid a_{i} \in \mathbb{R}, a_{i} \geq 0\right\},
$$

and the set $\left\{v_{1}, \cdots, v_{k}\right\}$ is a subset of some $\mathbb{Z}$-basis of $N$. In this case, we call $v_{1}, \cdots, v_{k} \in N$ the integral generators of $\sigma$.

Definition 2.2. A regular cone $\sigma^{\prime}$ is called a face of a regular cone $\sigma$ (we write $\left.\sigma^{\prime} \prec \sigma\right)$ if the set of integral generators of $\sigma^{\prime}$ is a subset of the set of integral generators of $\sigma$.

Definition 2.3. A finite system $\Sigma=\sigma_{1}, \cdots, \sigma_{s}$ of regular cones in $N_{\mathbb{R}}$ is called a complete $n$-dimensional fan of regular cones, if the following conditions are satisfied.

1. if $\sigma \in \Sigma$ and $\sigma^{\prime} \prec \sigma$, then $\sigma^{\prime} \in \Sigma$;

2. if $\sigma, \sigma^{\prime}$ are in $\Sigma$, then $\sigma^{\prime} \cap \sigma \prec \sigma$ and $\sigma^{\prime} \cap \sigma \prec \sigma^{\prime}$;

3. $N_{\mathbb{R}}=\sigma_{1} \cup \cdots \cup \sigma_{s}$.

The set of all $k$-dimensional cones in $\Sigma$ will be denoted by $\Sigma^{(k)}$.

EXAMPLE 2.4. Consider basis vectors $e_{1}, \cdots, e_{n}$ in a $n$-dimensional real vector space. Let $v_{i}=e_{i}$ for $i=1, \cdots, n$ and let $v_{n+1}=-e_{1}-e_{2}-\cdots-e_{n}$. Any $k$ element subset $I \subset\left\{v_{1}, \cdots, v_{n+1}\right\}$ for $(k \leq n)$ generates a $k$-dimensional regular cone $\sigma(I)$. The set $\Sigma(n)$ consisting of $2^{n+1}-1$ cones $\sigma(I)$ generated by $I$ is a complete $n$-dimensional fan of regular cones, with which later we will associate a projective space $\mathbb{P}^{n}$.

Definition 2.5. Let $\Sigma$ be a complete $n$-dimensional fan of regular cones. Denote by $G(\Sigma)=\left\{v_{1}, \cdots, v_{N}\right\}$ the set of all generators of 1-dimensional cones in $\Sigma(N=$ Card $\left.\Sigma^{(1)}\right)$. We call a subset $\mathcal{P}=\left\{v_{i_{1}}, \cdots, v_{i_{p}}\right\} \subset G(\Sigma)$ a primitive collection if $\left\{v_{i_{1}}, \cdots, v_{i_{p}}\right\}$ does not generate $p$-dimensional cone in $\Sigma$, while for all $k(0 \leq k<p)$ each $k$-element subset of $\mathcal{P}$ generates a $k$-dimensional cone in $\Sigma$.

EXAMPLE 2.6. Let $\Sigma$ be a fan from Example 2.4. Then there exists the unique primitive collection $\mathcal{P}$ which is the set of all generators $\left\{v_{1}, \cdots, v_{n+1}\right\}$.

Definition 2.7. Let $\mathbb{C}^{N}$ be $N$-dimensional affine space over $\mathbb{C}$ with the set of coordinates $z_{1}, \cdots, z_{N}$ which are in the one-to-one correspondence $z_{i} \leftrightarrow v_{i}$ with elements of $G(\Sigma)$. Let $\mathcal{P}=\left\{v_{i_{1}}, \cdots, v_{i_{p}}\right\}$ be a primitive collection in $G(\Sigma)$. Denote by $\mathbb{A}(\mathcal{P})$ the $(N-p)$-dimensional affine subspace in $\mathbb{C}^{n}$ defined by the equations

$$
z_{i_{1}}=\cdots=z_{i_{p}}=0
$$


REMARK 2.8. Since every primitive collection $\mathcal{P}$ has at least two elements, the codimension of $\mathbb{A}(\mathcal{P})$ is at least 2 .

Definition 2.9. Define the closed algebraic subset $Z(\Sigma)$ in $\mathbb{C}^{N}$ as follows

$$
Z(\Sigma)=\cup_{\mathcal{P}} \mathbb{A}(\mathcal{P}),
$$

where $\mathcal{P}$ runs over all primitive collections in $G(\Sigma)$. Put

$$
U(\Sigma)=\mathbb{C}^{N} \backslash Z(\Sigma)
$$

DeFinition 2.10. Let $\mathbb{K}$ be the subgroup in $\mathbb{Z}^{N}$ consisting of all lattice vectors $\lambda=\left(\lambda_{1}, \cdots, \lambda_{N}\right)$ such that

$$
\lambda_{1} v_{1}+\cdots+\lambda_{N} v_{N}=0
$$

Obviously $\mathbb{K}$ is isomorphic to $\mathbb{Z}^{N-n}$ and we have the exact sequence:

$$
0 \rightarrow \mathbb{K} \rightarrow \mathbb{Z}^{N} \stackrel{\pi}{\rightarrow} \mathbb{Z}^{n} \rightarrow 0,
$$

where the map $\pi$ sends the basis vectors $e_{i}$ to $v_{i}$ for $i=1, \cdots, N$.

Definition 2.11. Let $\Sigma$ be a complete $n$-dimensional fan of regular cones. Define $D(\Sigma)$ to be the connected commutative subgroup in $\left(\mathbb{C}^{*}\right)^{N}$ generated by all oneparameter subgroups

$$
\begin{aligned}
& a_{\lambda}: \mathbb{C}^{*} \rightarrow\left(\mathbb{C}^{*}\right)^{N}, \\
& t \mapsto\left(t^{\lambda_{1}}, \cdots, t^{\lambda_{N}}\right)
\end{aligned}
$$

where $\lambda=\left(\lambda_{1}, \cdots, \lambda_{N}\right) \in \mathbb{K}$.

It is easy to see from the definition that $D(\Sigma)$ acts freely on $U(\Sigma)$. Now we are ready to give a definition of the compact toric manifold $X_{\sigma}$ associated with a complete n-dimensional fan of regular cones $\Sigma$.

DEFINITION 2.12. Let $\Sigma$ be a complete $n$-dimensional fan of regular cones. Then the quotient

$$
X_{\Sigma}=U(\Sigma) / D(\Sigma)
$$

is called the compact toric manifold associated with $\Sigma$.

EXAMPLE 2.13. Let $\Sigma$ be a fan $\Sigma(n)$ from Example 2.4. By 2.6, $U(\Sigma(n))=\mathbb{C}^{n+1} \backslash$ $\{0\}$. By the definition of $\Sigma(n)$, the subgroup $\mathbb{K}$ is generated by $(1, \cdots, 1) \in \mathbb{Z}^{n+1}$. Thus $D(\Sigma) \subset\left(\mathbb{C}^{*}\right)^{N}$ consists of the elements $(t, \cdots, t)$, where $t \in \mathbb{C}^{*}$. So the toric manifold associated with $\Sigma(n)$ is the ordinary $n$-dimensional projective space.

There exists a simple open coverings of $U(\Sigma)$ by affine algebraic varieties. 
Proposition 2.1. Let $\sigma$ be a k-dimensional cone in $\Sigma$ generated by $\left\{v_{i_{1}}, \cdots, v_{i_{k}}\right\}$. Define the open subset $U(\sigma) \subset \mathbb{C}^{N}$ as

$$
U(\sigma)=\left\{\left(z_{1}, \cdots, z_{N}\right) \in \mathbb{C}^{N} \mid z_{j} \neq 0 \text { for all } j \notin\left\{i_{1}, \cdots, i_{k}\right\}\right\} .
$$

Then the open sets $U(\sigma)$ have the following properties:

1.

$$
U(\Sigma)=\cup_{\sigma \in \Sigma} U(\sigma)
$$

2. if $\sigma \prec \sigma^{\prime}$, then $U(\sigma) \subset U\left(\sigma^{\prime}\right)$;

3. for any two cone $\sigma_{1}, \sigma_{2} \in \Sigma$, one has $U\left(\sigma_{1}\right) \cap U\left(\sigma_{2}\right)=U\left(\sigma_{1} \cap \sigma_{2}\right)$; in particular,

$$
U(\Sigma)=\bigcup_{\sigma \in \Sigma^{(n)}} U(\sigma)
$$

Proposition 2.2. Let $\sigma$ be an n-dimensional cone in $\Sigma^{(n)}$ generated by $\left\{v_{i_{1}}, \cdots, v_{i_{n}}\right\}$, which spans the lattice $N$. We denote the dual $\mathbb{Z}$-basis of the lattice $M$ by $\left\{u_{i_{1}}, \cdots, u_{i_{n}}\right\}$. i.e.

$$
\left\langle v_{i_{k}}, u_{i_{l}}\right\rangle=\delta_{k, l}
$$

where $\langle\cdot, \cdot\rangle$ is the canonical pairing between lattices $N$ and $M$.

Then the affine open subset $U(\sigma)$ is isomorphic to $\mathbb{C}^{n} \times\left(\mathbb{C}^{*}\right)^{N-n}$, the action of $D(\Sigma)$ on $U(\sigma)$ is free, and the space of $D(\Sigma)$-orbits is isomorphic to the affine space $U_{\sigma}=\mathbb{C}^{n}$ whose coordinate functions $x_{1}^{\sigma}, \cdots, x_{n}^{\sigma}$ are $n$ Laurent monomials in $z_{1}, \cdots, z_{N}$ :

$$
\left\{\begin{array}{c}
x_{1}^{\sigma}=z_{1}^{\left\langle v_{1}, u_{i_{1}}\right\rangle} \cdots z_{N}^{\left\langle v_{N}, u_{i_{1}}\right\rangle} \\
\vdots \\
x_{n}^{\sigma}=z_{1}^{\left\langle v_{1}, u_{i_{n}}\right\rangle} \cdots z_{N}^{\left\langle v_{N}, u_{i_{n}}\right\rangle}
\end{array}\right.
$$

The last statement yields a general formula for the local affine coordinates $x_{1}^{\sigma}, \cdots, x_{n}^{\sigma}$ of a point $p \in U_{\sigma}$ as functions of its "homogeneous coordinates" $z_{1}, \cdots, z_{N}$.

3. Symplectic forms of toric manifolds. In the last section, we associated a compact manifold $X_{\Sigma}$ to a fan $\Sigma$. In this section, we review the construction of symplectic (Kähler) manifold associated to a convex polytope $P$.

Let $M$ be a dual lattice, we consider a convex polytope $P$ in $M_{\mathbb{R}}$ defined by

$$
\left\{x \in M_{\mathbb{R}} \mid\left\langle x, v_{j}\right\rangle \geq \lambda_{j} \text { for } j=1, \cdots, N\right\}
$$

where $\langle\cdot, \cdot\rangle$ is a dot product of $M_{\mathbb{R}} \cong \mathbb{R}^{n}$. Namely, $v_{j}$ 's are inward normal vectors to the codimension 1 faces of the polytope $P$. We associate to it a fan in the lattice $N$ as follows: With any face $\Gamma$ of $P$, fix a point $m$ in the (relative) interior of $\Gamma$ and define

$$
\sigma_{\Gamma}=\cup_{r \geq 0} r \cdot(P-m) .
$$

The associated fan is the family $\Sigma(P)$ of dual convex cones

$$
\begin{aligned}
\check{\sigma}_{\Gamma} & =\left\{x \in N_{\mathbb{R}} \mid\langle y, x\rangle \geq 0 \quad \forall y \in \sigma_{\Gamma}\right\} \\
& =\left\{x \in N_{\mathbb{R}} \mid\langle m, x\rangle \leq\langle p, x\rangle \forall p \in P, m \in \Gamma\right\}
\end{aligned}
$$


where $\langle\cdot, \cdot\rangle$ is dual pairing $M_{\mathbb{R}}$ and $N_{\mathbb{R}}$. Hence we obtain a compact toric manifold $X_{\Sigma(P)}$ associated to a fan $\Sigma(P)$.

Now we define a symplectic (Kähler) form on $X_{\Sigma(P)}$ as follows. Recall the exact sequence :

$$
0 \rightarrow \mathbb{K} \stackrel{i}{\rightarrow} \mathbb{Z}^{N} \stackrel{\pi}{\rightarrow} \mathbb{Z}^{n} \rightarrow 0 .
$$

It induces another exact sequence :

$$
0 \rightarrow K \rightarrow \mathbb{R}^{N} / \mathbb{Z}^{N} \rightarrow \mathbb{R}^{n} / \mathbb{Z}^{n} \rightarrow 0 .
$$

Denote by $k$ the Lie algebra of the real torus $K$. Then we have the exact sequence of Lie algebras:

$$
0 \rightarrow k \rightarrow \mathbb{R}^{N} \stackrel{\pi}{\rightarrow} \mathbb{R}^{n} \rightarrow 0
$$

And we have the dual of above exact sequence:

$$
0 \rightarrow\left(\mathbb{R}^{n}\right)^{*} \rightarrow\left(\mathbb{R}^{N}\right)^{*} \stackrel{i^{*}}{\rightarrow} k^{*} \rightarrow 0 .
$$

Now, consider $\mathbb{C}^{N}$ with symplectic form $\frac{i}{2} \sum d z_{k} \wedge d \bar{z}_{k}$. The standard action $T^{n}$ on $\mathbb{C}^{n}$ is hamiltonian with moment map

$$
\mu\left(z_{1}, \cdots, z_{N}\right)=\frac{1}{2}\left(\left|z_{1}\right|^{2}, \cdots,\left|z_{N}\right|^{2}\right)
$$

For the moment map $\mu_{K}$ of the $K$ action is then given by

$$
\mu_{K}=i^{*} \circ \mu: \mathbb{C}^{N} \rightarrow k^{*} .
$$

If we choose a $\mathbb{Z}$-basis of $\mathbb{K} \subset \mathbb{Z}^{N}$ as

$$
Q_{1}=\left(Q_{11}, \cdots, Q_{N 1}\right), \cdots, Q_{k}=\left(Q_{1 k}, \cdots, Q_{N k}\right)
$$

and $\left\{q^{1}, \cdots, q^{k}\right\}$ be its dual basis of $\mathbb{K}^{*}$. Then the map $i^{*}$ is given by the matrix $Q^{t}$ and so we have

$$
\mu_{K}\left(z_{1}, \cdots, z_{N}\right)=\frac{1}{2}\left(\sum_{j=1}^{N} Q_{j 1}\left|z_{j}\right|^{2}, \cdots, \sum_{j=1}^{N} Q_{j k}\left|z_{j}\right|^{2}\right) \in \mathbb{R}^{k} \cong k^{*}
$$

in the coordinates associated to the basis $\left\{q^{1}, \cdots, q^{k}\right\}$. We denote again by $\mu_{K}$ the restriction of $\mu_{K}$ on $U(\Sigma) \subset \mathbb{C}^{N}$.

Proposition 3.1 (Audin [Au], Proposition 6.3.1.). Then for any $r=$ $\left(r_{1}, \cdots, r_{N-n}\right) \in \mu_{K}(U(\Sigma)) \subset k^{*}$, we have a diffeomorphism

$$
\mu_{K}^{-1}(r) / K \cong U(\Sigma) / D(\Sigma)=X_{\Sigma} .
$$

And for each (regular) value of $r \in k^{*}$, we can associate a symplectic form $\omega_{P}$ on the manifold $X_{\Sigma}$ by symplectic reduction $[\mathrm{MW}]$.

To obtain the original polytope $P$ that we started with, we need to choose $r$ as follows: Consider $\lambda_{j}$ for $j=1, \cdots, N$ which we used to define our polytope $P$ by the set of inequalities $\left\langle x, v_{j}\right\rangle \geq \lambda_{j}$. Then, for each $a=1, \cdots, N-n$, let

$$
r_{a}=-\sum_{j=1}^{N} Q_{j a} \lambda_{j}
$$


Then we have

$$
\mu_{K}^{-1}\left(r_{1}, \cdots, r_{N-n}\right) / K \cong X_{\Sigma(P)}
$$

and for the residual $T^{n} \cong T^{N} / K$ action on $X_{\Sigma(P)}$, and for its moment map $\mu_{T}$, we have

$$
\mu_{T}\left(X_{\Sigma(P)}\right)=P
$$

In fact, Guillemin $[\mathrm{Gu}]$ proved the following explicit closed formula for the Kähler form

Theorem 3.2 (Guillemin). Let $P, X_{\Sigma(P)}, \omega_{P}$ and

$$
\mu_{T}: X_{\Sigma(P)} \rightarrow\left(\mathbb{R}^{N} / k\right)^{*} \cong\left(\mathbb{R}^{n}\right)^{*}
$$

be the moment map defined as above. Define the functions on $\left(\mathbb{R}^{n}\right)^{*}$

$$
\begin{aligned}
\ell_{i}(x) & =\left\langle x, v_{i}\right\rangle-\lambda_{i} \text { for } i=1, \cdots, N \\
\ell_{\infty}(x) & =\sum_{i=1}^{N}\left\langle x, v_{i}\right\rangle=\left\langle x, \sum_{i=1}^{N} v_{i}\right\rangle .
\end{aligned}
$$

Then we have

$$
\omega_{P}=\sqrt{-1} \partial \bar{\partial} \mu_{T}^{*}\left(\sum_{i=1}^{N} \lambda_{i}\left(\log \ell_{i}\right)+\ell_{\infty}\right)
$$

on $\operatorname{int}(P)$.

4. Adapted Floer cohomology of the torus fibers. Let $\left(X_{\Sigma(P)}, \omega_{P}\right)$ be a $2 n$ dimensional symplectic toric manifold with $T^{n}$-action constructed from the polytope $P \subset M_{\mathbb{R}}$. Each $T^{n}$ orbit associated to an interior point in $P$ is a Lagrangian submanifold of $X_{\Sigma(P)}$. Such an orbit can be obtained as $\mu_{T}^{-1}(A)$ for $A \in \operatorname{int}\left(\mu_{T}\left(X_{\Sigma(P)}\right)\right)$ for the moment map $\mu_{T}$.

We fix one such orbit (non-singular) and denote it by $L$. In this paper, we will study the Floer cohomology of these Lagrangian tori and compute this by computing its Bott-Morse theory version $H F^{B M}\left(L ; J_{0}\right)$ as in [Cho1]. This is the $m_{1}$-homology of the $A_{\infty}$-algebra of Lagrangian submanifolds defined in [FOOO]. It will be shown that in this case, obstruction class, $m_{0}$, turned out to be non-zero, but is a multiple of fundamental class, which makes $m_{1}^{2}=0$ (See Theorem 7.1). Note that in this case we can set the bounding cochain $b=0$ (See [FOOO] for its defintion). The explicit computation of Bott-Morse Floer cohomology is given in section 10.

A natural question is its relation to the Floer cohomology groups $H F(L, \phi(L))$ for a Hamiltonian isotopy of $M$. One important difference between the Clifford torus and the general torus fibers is that the former is monotone [O1], [O4] while the latter are not. Since the obstruction classes defined in [FOOO] do not vanish for the Lagrangian submanifold $L$, it is not clear whether the standard Floer cohomology $H F(L, \phi(L))$ is defined and invariant under the change of Hamiltonian isotopy, or whether it is isomorphic to the Bott-Morse version $H F^{B M}\left(L ; J_{0}\right)$ when $L$ is not monotone.

In this section, we will define a restricted version of the Floer cohomology which exploits some special geometry of Lagrangian torus fibers in the toric manifolds. We 
will call this adapted Floer cohomology and denote it by $H F^{a d}\left(L ; J_{0}\right)$. Important ingredients for the construction of the adapted Floer cohomology $H F^{a d}\left(L ; J_{0}\right)$ are the following three theorems whose proof will be postponed to the next two sections.

[Maslov index formula] For a symplectic toric manifold $X_{\Sigma(P)}$, let $L$ be a Lagrangian $T^{n}$ orbit. Then the Maslov index of any holomorphic disc with boundary lying on $L$ is twice the sum of intersection multiplicities of the image of the disc with the codimension 1 submanifolds $V\left(v_{j}\right)$ for $v_{j} \in \Sigma^{(1)}$ for all $j=1, \cdots, N$.

[Classification theorem] Let $\widetilde{L} \subset \mathbb{C}^{N} \backslash Z(\Sigma)$ be a fixed orbit of the real $N$-torus $\left(S^{1}\right)^{N}$. Any holomorphic map $w:\left(D^{2}, \partial D^{2}\right) \rightarrow\left(X_{\Sigma(P)}, L\right)$ can be lifted to a holomorphic map

$$
\widetilde{w}:\left(D^{2}, \partial D^{2}\right) \rightarrow\left(\mathbb{C}^{N} \backslash Z(\Sigma), \widetilde{L}\right)
$$

so that each homogeneous coordinates functions $z_{1}(\widetilde{w}), \cdots, z_{N}(\widetilde{w})$ are given by Blaschke products with constant factors.

$$
\text { i.e. } z_{j}(\widetilde{w})=c_{j} \cdot \prod_{k=1}^{\mu_{j}} \frac{z-\alpha_{j, k}}{1-\bar{\alpha}_{j, k} z}
$$

for $c_{j} \in \mathbb{C}^{*}$ and non-negative integers $\mu_{j}$ for each $j=1, \cdots, N$. In particular, there is no non-constant holomorphic discs of non-positive Maslov indices.

[Regularity theorem] The discs in the classification theorem are Fredholm regular, i.e., its linearization map is surjective.

Assuming these theorems for the moment, we proceed construction of $H F^{a d}\left(L ; J_{0}\right)$ of $\left(L ; J_{0}\right)$. We denote the standard integrable complex structure on $X$ by $J_{0}$. Let $\phi$ be a Hamiltonian diffeomorphism such that $\phi(L)$ intersects $L$ transversely. Let $\mathcal{J}_{\omega}(X)$ be the set of almost complex structures of $X$ compatible to $\omega$. We consider the set of paths $J^{\prime}:[0,1] \rightarrow \mathcal{J}_{\omega}(X)$ with

$$
J^{\prime}(0)=J_{0}, \quad J^{\prime}(1)=\phi_{*} J_{0}
$$

denote it by $j_{\left(\phi, J_{0}\right)}$.

Similar theorems obviously hold for the pair $\left(\phi(L), \phi_{*} J_{0}\right)$ as for $\left(L ; J_{0}\right)$. In particular, there is no non-constant holomorphic discs of non-positive Maslov indices for the pair $\left(\phi(L), \phi_{*} J_{0}\right)$ either.

REMARK 4.1. The set $j_{\left(\phi, J_{0}\right)}$ was considered and played an important role in [O6] in relation to the formulation of Floer homology of Hamiltonian diffeomorphisms over the mapping torus of $\phi$. It appears that considering this set of paths depending on the triple $\left(L, J_{0} ; \phi\right)$ enable us to define the Floer homology of Lagrangian submanifolds in a more flexible way when the given pair $\left(L ; J_{0}\right)$ has some special structure of the moduli of $J_{0}$-holomorphic discs attached to $L$ as in our case.

Now we restrict to the paths $J^{\prime} \in j_{\left(\phi, J_{0}\right)}$ for the study of Floer's equations

$$
\left\{\begin{array}{l}
\frac{\partial u}{\partial \tau}+J_{t}^{\prime} \frac{\partial u}{\partial t}=0 \\
u(\tau, 0) \in L, u(\tau, 1) \in \phi(L)
\end{array}\right.
$$

in the definition of the Floer boundary operator. Now for given pair $x, y \in L \cap \phi(L)$, we study the moduli space

$$
\mathcal{M}\left(x, y ; J^{\prime}\right)
$$


for the Fredholm index $\mu(x, y)=0,1$ or 2 . It is easy to see that for a generic $J^{\prime} \in j_{\left(\phi, J_{0}\right)}$ the (uncompactified) moduli space $\mathcal{M}\left(x, y ; J^{\prime}\right)$ is transversal(See [O5]). The following proposition is the reason why we restrict $J^{\prime}$ to the ones coming from $j_{\left(\phi, J_{0}\right)}$.

Proposition 4.1. Assume $X_{\Sigma(P)}$ is Fano. Let $\phi$ be a Hamiltonian diffeomorphism such that $\phi(L)$ intersects $L$ transversely and choose a generic $J^{\prime} \in j_{\left(\phi, J_{0}\right)}$. Assume that $x, y \in L \cap \phi(L)$ with $\mu(x, y)=0,1$ or 2 . Then the following holds:

1. When $\mu(x, y)=0, \mathcal{M}\left(x, y ; J^{\prime}\right) / \mathbb{R}$ is empty.

2. When $\mu(x, y)=1, \mathcal{M}\left(x, y ; J^{\prime}\right) / \mathbb{R}$ is a compact manifold of dimension zero

3. When $\mu(x, z)=2$,

(a) if $x \neq z, \mathcal{M}\left(x, z ; J^{\prime}\right) / \mathbb{R}$ can be compactified into a compact manifold with boundary of dimension one, whose boundary consists of the form

$$
v_{1} \sharp v_{2}
$$

where $v_{1} \in \mathcal{M}\left(x, y ; J^{\prime}\right)$ and $v_{2} \in \mathcal{M}\left(y, z ; J^{\prime}\right)$.

(b) if $x=z, \mathcal{M}\left(x, x ; J^{\prime}\right)$ can be compactified into a compact manifold with boundary of dimension one, whose boundary consists of the types

$$
v_{1} \sharp v_{2}
$$

where $v_{i}$ 's are types either of (4.2) or that for which one of $v_{i}$ 's is constant and the other is a $J_{0}$-holomorphic disc with boundary lying on $L$ or a $\phi_{*}\left(J_{0}\right)$-holomorphic disc with boundary lying on $\phi(L)$.

Proof. First note that non-constant holomorphic discs with boundary on one of the Lagrangian submanifold, $L$ or $\phi(L)$, have positive Maslov indices (and so greater than or equal to 2). Once this is in our disposition, the proof of this fact follows by the dimension counting arguments from [O1], [O4]. We omit the details of the argument referring to [O1].

REMARK 4.2. Unlike the case [O1] or [O4] where we allow to vary the almost complex structures, since we prefer to keep the usage of integrable complex structure $J_{0}$, we also need to prove that the singular curves in part (3b) are also regular (or more precisely the relevant evaluation maps are transverse in forming the fiber products). This follows from the fact that $L$ is a torus orbit of the torus action on $X_{\Sigma(P)}$.

Corollary 4.2. Under the hypothesis as in Proposition 4.1, the Floer cohomology $\operatorname{HF}\left(L, \phi(L) ; J^{\prime}\right)$ is well-defined.

We can now compare two Floer cohomology $H F\left(L, \phi(L) ; J^{\prime}\right)$ with $J^{\prime} \in j_{\left(\phi, J_{0}\right)}$ and $\left.H F^{*}(L, \psi(L)) ; J^{\prime \prime}\right)$ with $J^{\prime \prime} \in j_{\left(\psi, J_{0}\right)}$ by considering paths

$$
\begin{aligned}
& \Phi=\left\{\phi^{s}\right\}_{0 \leq s \leq 1} ; \phi^{0}=\phi, \phi^{1}=\psi \\
& \bar{J}=\left\{J^{s}\right\}_{0 \leq s \leq 1} ; J^{0}=J^{\prime}, J^{1}=J^{\prime \prime}, J^{s} \in j_{\left(\phi^{s}, J_{0}\right)}
\end{aligned}
$$

and the continuity equation

$$
\left\{\begin{array}{l}
\frac{\partial u}{\partial \tau}+J_{t}^{\rho(\tau)} \frac{\partial u}{\partial t}=0 \\
u(\tau, 0) \in L, u(\tau, 1) \in \phi^{\rho(\tau)}(L)
\end{array}\right.
$$


where $\rho: \mathbb{R} \rightarrow[0,1]$ is a monotonically increasing function

$$
\rho= \begin{cases}0 & \text { for } \tau \leq-R \\ 1 & \text { for } \tau \geq R\end{cases}
$$

for some sufficiently large $R>0$. Again by the same reasoning using the choice $J^{s} \in j_{\left(\phi^{s}, J_{0}\right)}$, we can prove that the continuity equation defines a chain map

$$
h_{(\Phi, \bar{J})}: C F\left(L, \phi(L) ; \delta_{J^{\prime}}\right) \rightarrow C F\left(L, \psi(L) ; \delta_{J^{\prime \prime}}\right)
$$

which is an isomorphism. We refer to [O1], [O4] for the proof in the monotone case, which obviously generalizes in the current Fano toric case if we use the set-up of the adapted Floer cohomology. More specifically we use the special property of the pair $\left(L, J_{0}\right)$ mentioned in the three theorems in the beginning of this section. This proves the well-definedness and the invariance property of $H F\left(L, \phi(L) ; J^{\prime}\right)$. We denote the canonical isomorphism class of $H F\left(L, \phi(L) ; J^{\prime}\right)$ over $\phi$ and $J^{\prime} \in j_{\left(\phi, J_{0}\right)}$ by $H F^{a d}\left(L ; J_{0}\right)$.

We will compute this group by computing the Bott-Morse version of the Floer cohomology, which we denote by $H F^{B M}\left(L ; J_{0}\right)$. Because the above structure theorems, this latter Floer cohomology group is well-defined. The following theorem permits us to do this for the computation of $H F\left(L, \phi(L) ; J^{\prime}\right)$.

Theorem 4.3. Assume $X_{\Sigma(P)}$ is Fano and let $L$ and $\phi, J^{\prime}$ as above. Then $H F^{B M}\left(L ; J_{0}\right)$ is well-defined and isomorphic to $H F^{a d}\left(L ; J_{0}\right)$. More specifically, $H F^{B M}\left(L ; J_{0}\right)$ is isomorphic to $H F\left(L, \phi(L) ; J^{\prime}\right)$ for any Hamiltonian diffeomorphism $\phi$ with $L$ intersection $\phi(L)$ transversely and a path $J^{\prime} \in j_{\left(\phi, J_{0}\right)}$.

Proof. The well-definedness of $H F^{B M}\left(L ; J_{0}\right)$ follows from the classification theorem which in particular implies that all holomorphic discs have positive Maslov indices and are regular, by examining the details of the construction from [section 7, FOOO].

For the second statement, it is enough to consider the case when $\phi$ is $C^{2}$-close to the identity. We refer to [O4] for the proof in the monotone case which obviously generalizes to the semi-positive case, in particular the Fano case $(X, \omega)$. A complete proof will be given in the revision of [FOOO].

REMARK 4.3 . Here we use the canonical complex structure $J_{0}$ and directly verify that the Floer cohomology $\operatorname{HF}^{B M}\left(L ; J_{0}\right)$ is well-defined. Once this is done, the general theory from [FOOO] (2006) implies that the Floer homology is defined and invariant under the Hamiltonian isotopy, as long as either the Bott-Morse case with time-independent $J$ or the adapted Floer cohomology are considered. This general result is not used in this paper. We refer interested readers to [FOOO] for the detailed explanations.

Having Theorem 4.3 in mind, we will compute the Bott-Morse Floer cohomology group $H F^{B M}\left(L ; J_{0}\right)$ in the rest of the paper.

5. Index formula and the classification of holomorphic discs. In this section, we will prove the Maslov index formula and the classification theorem mentioned in section 4. Let us first recall the definition of the Maslov index in this setting. If $w:\left(D^{2} \partial D^{2}\right) \rightarrow(X, L)$ is a smooth map of pairs, we can find a unique trivialization (up to homotopy) of the pull-back bundle $w^{*} T X \cong D^{2} \times \mathbb{C}^{n}$. This trivialization 
defines a map from $S^{1}=\partial D^{2}$ to $\Lambda\left(\mathbb{C}^{n}\right)=$ the set of lagrangian planes in $\mathbb{C}^{n}$, where there is a well-known Maslov class $\mu \in H^{1}\left(\Lambda\left(\mathbb{C}^{n}\right), \mathbb{Z}\right)$; see $[\mathrm{Ar}]$. We define

$$
I_{\mu, L}(w):=\mu\left(\partial D^{2}\right) \in \mathbb{Z} .
$$

For the smooth maps $\tilde{w}:(\Sigma, \partial \Sigma) \rightarrow(X, L)$ from the Riemann surface with boundary, one can define its Maslov index similarly by taking the sum of Maslov indices along $\partial \Sigma$ using the fact that symplectic vector bundle $\tilde{w}^{*} T X$ is always trival.

Before we state the theorem, we recall that for each generator $v_{j} \in \Sigma^{(1)}$, there is associated a codimension 1 subvariety $V\left(v_{j}\right)$. For the principle bundle $(U(\Sigma) \stackrel{\pi}{\rightarrow}$ $\left.X_{\Sigma(P)}\right), \pi^{-1}\left(V\left(v_{j}\right)\right)$ is defined by the equation $z_{j}=0$ in $U(\Sigma)$.

TheOrem 5.1 (The Maslov index formula). For a symplectic toric manifold $X_{\Sigma(P)}$, let $L$ be a Lagrangian $T^{n}$ orbit. Then the Maslov index of any holomorphic disc with boundary lying on $L$ is twice the sum of intersection multiplicities of the image of the disc with the codimension 1 submanifolds $V\left(v_{j}\right)$ for $v_{j} \in \Sigma^{(1)}$ for all $j=1, \cdots, N$.

Proof. As in [Cho1], we deform a holomorphic disc $w:\left(D^{2}, \partial D^{2}\right) \rightarrow(X, L)$ near the intersections with $V\left(v_{j}\right)$ 's. It is easy to see that the intersections are discrete and there are only finitely many of them because of holomorphicity of the map $w$. Denote by $p_{1} \in D^{2}$ a point in the preimage of the intersection. i.e. $p_{1} \in w^{-1}$ (image $(w) \cap$ $V\left(v_{j}\right)$ ) for some $j$. We describe how to deform $w$ as a smooth map near the point $p_{1}$ and such deformation will be carried out near every preimages of intersections.

Note that $w\left(p_{1}\right)$ may lie in the intersection of several $V\left(v_{j}\right)$ 's: Denote them by $V\left(v_{i_{1}}\right), \cdots, V\left(v_{i_{k}}\right)$. Then, We have

$$
w(p) \in V\left(v_{i_{1}}\right) \cap \cdots \cap V\left(v_{i_{k}}\right)
$$

The fact that $V\left(v_{i_{1}}\right) \cap \cdots \cap V\left(v_{i_{k}}\right) \neq 0$ implies that $\left\{v_{i_{1}}, \cdots, v_{i_{k}}\right\}$ is not a primitive collection (See Definition 2.5). Since the fan $\Sigma$ is complete, we may choose $(n-k)$ generators $v_{i_{k+1}}, \cdots, v_{i_{n}}$ so that $\left\langle v_{i_{1}}, \cdots, v_{i_{n}}\right\rangle$ defines a $n$-dimensional cone $\sigma$ in $\Sigma$.

We may consider the map $w$ near $p_{1}$ as a map into the affine open set $\mathbb{C}^{n}=$ $\operatorname{Spec}(\check{\sigma} \cap M)$ as in [Ful]. More precisely, the coordinate functions of this affine open set $\mathbb{C}^{n}$ is given as in Proposition 2.2. Denote by $d_{1}, \cdots, d_{k}$ the intersection multiplicities of the map $w$ with $V\left(v_{i_{1}}\right), \cdots, V\left(v_{i_{k}}\right)$. In other words, if we represent the map $w$ in terms of homogeneous coordinates, then the homogeneous coordinate functions $z_{i_{1}}, \cdots, z_{i_{k}}$ will have order of zero $d_{1}, \cdots, d_{k}$ at $p_{1}$ and other homogeneous coordinate functions are non-vanishing near $p_{1}$.

As in Proposition 2.2, let $\left\{u_{i_{1}}, \cdots, u_{i_{n}}\right\}$ be the basis of $M$ dual to $\left\{v_{i_{1}}, \cdots, v_{i_{n}}\right\}$.

$$
\left\langle u_{i_{j}}, v_{i_{k}}\right\rangle=\delta_{j, k}
$$

Then, the affine coordinate function $x_{1}^{\sigma}$ is

$$
\begin{aligned}
x_{1}^{\sigma} & =z_{1}^{\left\langle v_{1}, u_{i_{1}}\right\rangle} \cdots z_{N}^{\left\langle v_{N}, u_{i_{1}}\right\rangle} \\
& =C(z) \cdot z_{i_{1}}^{\left\langle v_{i_{1}}, u_{i_{1}}\right\rangle} \\
& =C(z) \cdot z_{i_{1}}
\end{aligned}
$$

where $C(z)$ is a function nonvanishing near $p_{1}$. Therefore, the affine coordinate function $x_{1}^{\sigma}$ has order of zero $d_{1}$ at $p_{1}$. Similarly, $x_{2}^{\sigma}, \cdots, x_{k}^{\sigma}$ have order of zero 
$d_{2}, \cdots, d_{k}$ at $p_{1}$. For $j>k, x_{j}^{\sigma}$ is non-vanishing near $p_{1}$. We may further assume that $p_{1}=0 \in D^{2}$. Then, the map $w$ near $p_{1}$ can be written in affine coordinates as $\left(a_{1} z^{d_{1}}+\mathcal{O}\left(z^{d_{1}+1}\right), \cdots, a_{k} z^{d_{k}}+\mathcal{O}\left(z^{d_{k}+1}\right), a_{k+1}+\mathcal{O}(z), \cdots, a_{n}+\mathcal{O}(z)\right)$.

Now we are in the same situation as in [Cho1] Theorem 9.1. From now on, we will only sketch the arguments and refer readers to [Cho1] for details.

We label by $p_{2}, \cdots, p_{m} \in D^{2}$ all the other points whose image intersect with $V\left(v_{j}\right)$ for some $j$. We find disjoint open balls $B_{\epsilon}\left(p_{1}\right) \subset D^{2}$ centered at $p_{i}$ with fixed radius $\epsilon$ for sufficiently small $\epsilon$ for all $i=1,2, \cdots, m$.

Now we smoothly deform the map $w$ inside the ball $B_{\epsilon}\left(p_{1}\right)$, so that the deformed map $\widetilde{w}$ satisfies

$$
\left.\widetilde{w}\right|_{\partial B_{\epsilon / 2}\left(p_{1}\right)} \subset L
$$

and as a map into the affine open set $\mathbb{C}^{n}$ near $p_{1}$, the map $\widetilde{w}$ on $B_{\epsilon / 2}\left(p_{1}\right)$ is given by

$$
\left(\frac{a_{1} z^{d_{0}}}{\left|a_{0}\right|\left(\frac{\epsilon}{2}\right)^{d_{1}}}, \cdots, \frac{a_{k} z^{d_{k}}}{\left|a_{k}\right|\left(\frac{\epsilon}{2}\right)^{d_{k}}}, \frac{a_{k+1}}{\left|a_{k+1}\right|}, \cdots, \frac{a_{n}}{\left|a_{n}\right|}\right) .
$$

We perform the same kind of deformations for $p_{2}, p_{3}, \cdots, p_{m}$ inside the ball $B_{\epsilon}\left(p_{2}\right), \cdots, B_{\epsilon}\left(p_{m}\right)$ and write the resulting map as $\widetilde{w}$. Over the punctured disc

$$
\Sigma=D^{2} \backslash\left(B_{\epsilon}\left(p_{1}\right) \cup \cdots B_{\epsilon}\left(p_{m}\right)\right),
$$

the deformed map $\widetilde{w}$ does not intersect with the hyperplanes, and it intersects with the Lagrangian torus $L$ along the boundaries of the punctured disc.

Since the Maslov index is a homotopy invariant, we have $\mu(w)=\mu(\widetilde{w})$. Hence, we may compute the Maslov index of the map $\widetilde{w}$. Note that the boundary $\partial \Sigma$ is $\partial D^{2} \cup\left(\cup_{i} \partial B_{\epsilon / 2}\left(p_{i}\right)\right)$.

Since the image of the map $\widetilde{w}$ on the boundaries of the balls $B_{\epsilon / 2}\left(p_{i}\right)^{\prime} s$ lies on the Lagrangian submanifold $L$, the map $\widetilde{w}:(\Sigma, \partial \Sigma) \rightarrow(X, L)$ satisfies the Lagrangian boundary condition. Furthermore, since every intersection with the hyperplane occurs inside the balls $B_{\epsilon / 2},\left.\widetilde{w}\right|_{\Sigma}$ does not meet the hyperplanes. Hence, it can be considered as a map into the cotangent bundle of $L$, (If we take out all such codimension 1 submanifolds $V\left(v_{j}\right)$ 's from $X_{\Sigma(P)}$, there remains $\left(\mathbb{C}^{*}\right)^{n}$ which can be considered as the cotangent bundle of the torus orbit $L)$. Therefore we have

$$
\mu\left(\left.\widetilde{w}\right|_{\Sigma}\right)=0
$$

On the other hand, the Maslov index of the map $\left.\widetilde{w}\right|_{\Sigma}$ is given by the sum of the Maslov indices along $\partial \Sigma$ after fixing the trivialization.

Now consider the map $\widetilde{w}: D^{2} \rightarrow X$ and we fix a trivialization $\Phi$ of the pullback bundle $\widetilde{w}^{*} T X$. It gives a trivialization $\Phi_{\Sigma}$ of the pull-back bundle $\left(\left.\widetilde{w}\right|_{\Sigma}\right)^{*} T X$ restricted over $\Sigma$. In this trivialization, it is easy to see that

$$
\mu\left(\Phi_{\Sigma}, \partial D^{2}\right)=\mu\left(\Phi, \partial D^{2}\right)=\mu(\widetilde{w})=\mu(w) .
$$

Since the boundary of the balls $B_{\epsilon / 2}$ are oriented in the opposite way, and from the explicit description (5.3) of the deformed map on the ball $B_{\epsilon / 2}\left(p_{i}\right)$, we have

$$
\mu\left(\Phi_{\Sigma}, \partial B_{\epsilon / 2}\left(p_{i}\right)\right)=-2\left(\text { sum of intersection multiplicities in } B_{\epsilon / 2}\left(p_{i}\right)\right) .
$$

From the equation (5.4), we have

$$
\mu(w)-2(\text { sum of intersection multiplicities })=0 .
$$


Now, we use this index formula to classify all holomorphic discs with boundary lying on $L$. It is much easier if we describe them in terms of "homogeneous coordinates" of toric varieties. Namely we claim that homogeneous coordinate functions of any holomorphic discs with boundary on $L$ can be written as just Blaschke products with constant coefficients.

We recall that the homogeneous coordinates come from $\mathbb{C}^{N} \backslash Z(\Sigma)$ in Definition 2.9 and $\left(\mathbb{C}^{N} \backslash Z(\Sigma)\right) / D(\Sigma)=X_{\Sigma(P)}$. We denote by

$$
\begin{aligned}
& p_{\mathbb{C}}:\left(\mathbb{C}^{N}\right)^{*} \rightarrow\left(\mathbb{C}^{N}\right)^{*} / D(\Sigma) \cong\left(\mathbb{C}^{*}\right)^{n} \\
& \left.p_{T}:\left(T^{N}\right)^{*} \rightarrow\left(T^{N}\right) / T(\Sigma)\right) \cong T^{n}
\end{aligned}
$$

the natural projections.

ThEOREM 5.2 (Classification theorem). Let $\widetilde{L} \subset \mathbb{C}^{N} \backslash Z(\Sigma)$ be a fixed orbit of the real $N$-torus $\left(S^{1}\right)^{N}$. Any holomorphic map $w:\left(D^{2}, \partial D^{2}\right) \rightarrow\left(X_{\Sigma(P)}, L\right)$ can be lifted to a holomorphic map

$$
\widetilde{w}:\left(D^{2}, \partial D^{2}\right) \rightarrow\left(\mathbb{C}^{N} \backslash Z(\Sigma), \widetilde{L}\right)
$$

so that each homogeneous coordinates functions $z_{1}(\widetilde{w}), \cdots, z_{N}(\widetilde{w})$ are given by the Blaschke products with constant factors: i.e.,

$$
z_{j}(\widetilde{w})=c_{j} \cdot \prod_{k=1}^{\mu_{j}} \frac{z-\alpha_{j, k}}{1-\bar{\alpha}_{j, k} z}
$$

for $c_{j} \in \mathbb{C}^{*}$ and non-negative integers $\mu_{j}$ for each $j=1, \cdots, N$. And two such liftings $\widetilde{w}, \widetilde{w}^{\prime}$ of $w$ are related by

$$
\widetilde{w}^{\prime}=t \cdot \widetilde{w}
$$

where $t \in T(\Sigma)$ is a constant element in the real torus $T(\Sigma) \subset D(\Sigma)$.

Proof. Suppose the map $w$ meets the submanifold $V\left(v_{1}\right)$ at $w(\alpha)$ for $\alpha \in \operatorname{int}\left(D^{2}\right)$. We multiply the torus element

$$
p_{\mathbb{C}}\left(\left(\frac{1-\bar{\alpha} z}{z-\alpha}, 1,1, \cdots, 1\right)\right) \in\left(\mathbb{C}^{*}\right)^{N} / D(\Sigma) \cong\left(\mathbb{C}^{*}\right)^{n}
$$

to $w$ and denote the modified map by $w_{1}$. Note that the map $w_{1}$ still satisfies the boundary condition because we multiplied the element $(5.6)$ of the torus $\left(\mathbb{C}^{*}\right)^{N} / D(\Sigma)$ and the $L$ is an orbit thereof and $\left|\frac{1-\bar{\alpha} z}{z-\alpha}\right|=1$ on $\partial D^{2}$. And the intersection multiplicity of $w_{1}$ with $V\left(v_{1}\right)$ is one less than that of $w$.

By repeating the process, we may assume that we obtain a map $w_{d}$ which does not meet $V\left(v_{1}\right)$. Repeat the process for each $V\left(v_{j}\right)$ for $j=1, \cdots, N$. Hence we obtain a holomorphic map $w^{\prime}:\left(D^{2}, \partial D^{2}\right) \rightarrow(X, L)$ does not meet any codimension 1 submanifolds $V\left(v_{j}\right)$ 's. This map has Maslov index 0 and is contained in any affine open sets $\mathbb{C}^{n}$ of toric variety. It is easy to see that this map is indeed constant. Due to the boundary condition, this last map $w^{\prime}$ must be the constant map

$$
w^{\prime} \equiv x_{0}
$$


for some point $x_{0} \in L \subset X_{\Sigma(P)}$. We choose any point

$$
\left(c_{1}, \cdots, c_{N}\right) \in \pi^{-1}\left(x_{0}\right) \cap \widetilde{L}
$$

and then define $\widetilde{w}: D^{2} \rightarrow \mathbb{C}^{N}$ by the map whose $j$-th component is given by the formula

$$
z_{j}(\widetilde{w})=c_{j} \cdot \prod_{k=1}^{\mu_{j}} \frac{z-\alpha_{j, k}}{1-\bar{\alpha}_{j, k} z}
$$

where $\prod_{k=1}^{\mu_{j}} \frac{z-\alpha_{j, k}}{1-\bar{\alpha}_{j, k} z}$ is the reciprocal to the factors $\frac{1-\overline{\alpha_{j, k}} z}{z-\alpha_{j, k}}$ multiplied to cancel the intersection of $w$ with the divisor $V\left(v_{j}\right)$ in the above process.

Hence, we may deduce that homogeneous coordinates of any holomorphic disc can be written as Blaschke products. The map $\widetilde{w}$ obviously maps $\partial D^{2}$ to $\widetilde{L}$ since we made the choice $(5.7)$ for $\left(c_{0}, \cdots, c_{N}\right)$ and so $\widetilde{L}$ is a fixed $T^{N}$-orbit $\left(c_{0}, \cdots, c_{N}\right)$, and all the Blaschke factor has unit norm on $\partial D^{2}$. This proves the first part of the theorem.

For the second statement, we note that in the above formula (5.5) the data $\alpha_{j, k}$ and $\mu_{j}$ are completely determined by $w$ but not on the lifting, the coordinates functions of $\widetilde{w}^{\prime}(z)$ and $\widetilde{w}(z)$ have common zeros and poles. Therefore the ratios

$$
\frac{\widetilde{w}_{j}^{\prime}(z)}{\widetilde{w}_{j}(z)}
$$

defines a non-zero holomorphic function. Therefore if we define

$$
g_{j}=\frac{\widetilde{w}_{j}^{\prime}}{\widetilde{w}_{j}}, \quad g=\left(g_{1}, \cdots, g_{N}\right) \subset D(\Sigma) \subset\left(\mathbb{C}^{*}\right)^{N},
$$

$g$ defines a well-defined holomorphic map from $D^{2}$ to $D(\Sigma) \subset\left(\mathbb{C}^{*}\right)^{N}$ each of whose components has the unit norm on $\partial D^{2}$. Since $(D(\Sigma), T(\Sigma))$ is holomorphically isomorphic to the pair $\left(\left(\mathbb{C}^{*}\right)^{N-n}, T^{N-n}\right)$, the argument in the first part proves that $g$ must be constant.

This finishes the proof.

REMARK 5.1.

1. In the case of $\mathbb{P}^{N}$, a similar formula was proved in [Cho1].

2. The Maslov index of $w$ is $\sum_{j=1}^{N} \mu_{j}$ by Theorem 5.1.

6. Fredholm regularity of discs. In this section, we justify the use of the standard complex structure in the computation of the Floer cohomology. In general, to make a good moduli space of $J$-holomorphic discs $\mathcal{M}(X, L, \beta)$ is very difficult. It is especially so since all perturbations of the moduli spaces had to be compatible for all homotopy classes of the $J$-holomorphic discs. (See [FOOO] for details). But, we will show that the moduli space of holomorphic discs in toric Fano manifolds with boundary on torus fibers behaves rather nicely.

First we show the Fredholm regularity of the holomorphic discs. This implies that the moduli space of holomorphic discs (before compatification) are smooth manifolds of expected dimensions.

Theorem 6.1 (Regularity theorem). The discs in Theorem 5.2 are Fredholm regular. 
Proof. We have only to prove the surjectivity of the linearization of $\bar{\partial}$ at each holomorphic disc. Since the standard complex structure is integrable, linearized operator $D_{w}$ for a holomorphic disc $w$ is complex linear and exactly the Dolbeault derivative $\bar{\partial}$. And we will prove the surjectivity of this linearized operator.

We first recall the exact sequence

$$
0 \rightarrow \mathbb{K} \rightarrow \mathbb{Z}^{N} \stackrel{\pi}{\rightarrow} \mathbb{Z}^{n} \rightarrow 0 .
$$

This induces the exact sequence of the complex vector space

$$
0 \rightarrow \mathbb{C}^{\mathbb{K}} \rightarrow \mathbb{C}^{N} \stackrel{\pi}{\rightarrow} \mathbb{C}^{n} \rightarrow 0
$$

via tensoring with $\mathbb{C}$ where $\mathbb{C}^{\mathbb{K}}$ is the $N-n$ dimensional subspace of $\mathbb{C}^{N}$ spanned by $\mathbb{K} \subset \mathbb{Z}^{N}$. Note that this exact sequence is equivariant under the natural actions by the associated complex tori.

Now we explain implication of the existence of the above equivariant exact sequence on the study of Fredholm property of holomorphic map

$$
w:\left(D^{2}, \partial D^{2}\right) \rightarrow(X, L)
$$

where $L \subset X$ is a torus fiber $L=\mu^{-1}(\eta), \eta \in P \subset M_{\mathbb{R}}$.

We first need some general discussion on the sheaf of holomorphic sections of bundle pairs $(E, F)$ where $E$ is a complex vector bundle over $D^{2}$ and $F$ a real vector bundle over $\partial D^{2}$ such that $F \otimes \mathbb{C}$ is identified with $\left.E\right|_{\partial D^{2}}$. We denote by $(\mathcal{E}, \mathcal{F})$ the sheaf of holomorphic sections of $E$ with boundary values lying in $F$. We will be interested in the sheaf cohomology of $(\mathcal{E}, \mathcal{F})$ which we denote by

$$
H^{q}\left(D^{2}, \partial D^{2} ; E, F\right)=H^{q}(E, F) .
$$

Here the sheaf cohomology functors are the right derived functors of the global section functor from the category of sheaves of $\left(\mathcal{O}, \mathcal{O}_{\mathbb{R}}\right)$-modules on $D^{2}$ to the category of $\mathbb{R}$ modules, where $\left(\mathcal{O}, \mathcal{O}_{\mathbb{R}}\right)$ is the sheaf of holomorphic functions on $D^{2}$ with real boundary values. Denote by $\mathcal{A}^{0}(E, F)$ the sheaf of $C^{\infty}$ sections of $E$ with boundary values in $F$, and denote by $\mathcal{A}^{(0,1)}(E)$ the sheaf of $C^{\infty} E$-valued $(0,1)$-forms. The following is easy to check (see section 3.4 of [KL]).

Lemma 6.2. The sequence

$$
0 \rightarrow(\mathcal{E}, \mathcal{F}) \rightarrow \mathcal{A}^{0}(E, F) \stackrel{\bar{\partial}}{\rightarrow} \mathcal{A}^{0,1}(E) \rightarrow 0
$$

defines a fine resolution of $(\mathcal{E}, \mathcal{F})$.

Hence, the sheaf cohomology of $(\mathcal{E}, \mathcal{F})$ is given by the cohomology of the two term elliptic complex

$$
0 \rightarrow A^{0}(E, F) \stackrel{\bar{\partial}}{\rightarrow} A^{0,1}(E) \rightarrow 0,
$$

where $A^{0}(E, F)$ is the space of global $C^{\infty}$ sections of $E$ with boundary values in $F$, and $A^{0,1}(E)$ is the space of global $C^{\infty} E$-valued $(0,1)$-forms. From this, it follows that

$$
\begin{aligned}
& H^{0}(E, F) \cong \operatorname{ker} \bar{\partial} \\
& H^{1}(E, F) \cong \operatorname{coker} \bar{\partial} .
\end{aligned}
$$


Next let $(X, L)$ be a pair of Kähler manifold $X$ and a Lagrangian submanifold $L \subset X$. Consider a holomorphic disc $w:\left(D^{2}, \partial D^{2}\right) \rightarrow(X, L)$ and denote

$$
E=w^{*} T X, \quad F=(\partial w)^{*} T L .
$$

In terms of the sheaf cohomology group $H^{q}\left(D^{2}, \partial D^{2} ; E, F\right)$, the surjectivity of the linearization of the disc $w$ is equivalent to the vanishing result

$$
H^{1}\left(D^{2}, \partial D^{2} ; E, F\right)=\{0\} .
$$

Now we restrict to the case of our main interest as in Theorem 6.1. Let $w$ : $\left(D^{2}, \partial D^{2}\right) \rightarrow(X, L)$ be a holomorphic disc obtained in section 5 and $\widetilde{w}:\left(D^{2}, \partial D^{2}\right) \rightarrow$ $\left(\mathbb{C}^{N}, \widetilde{L}\right)$ be the lifting obtained in Theorem 5.2. From the expression of $\widetilde{w}$ in Theorem 5.2 , it follows that $\widetilde{w}\left(\partial D^{2}\right)$ is contained in a torus orbit of $\left(S^{1}\right)^{N}$

$$
\widetilde{L}=\left(S^{1}\right)^{N} \cdot\left(c_{1}, \cdots, c_{N}\right) \subset \pi^{-1}(L) \subset \mathbb{C}^{N} .
$$

We denote by

$$
\begin{aligned}
(E, F) & =\left(w^{*} T X,(\partial w)^{*} T L\right) \\
(\widetilde{E}, \widetilde{F}) & \left.=\left(D^{2} \times \mathbb{C}^{N},(\partial \widetilde{w})^{*}(T \widetilde{L})\right)\right) \\
\left(E_{\mathbb{K}}, F_{\mathbb{K}}\right) & =\left((\widetilde{w})^{*}\left(\operatorname{TOrb}_{\left(\mathbb{C}_{*}\right)^{\mathbb{K}}}\right),(\partial \widetilde{w})^{*}\left(\operatorname{TOrb}_{K}\right)\right)
\end{aligned}
$$

and by

$$
(\mathcal{E}, \mathcal{F}), \quad(\widetilde{\mathcal{E}}, \widetilde{\mathcal{F}}), \quad\left(\mathcal{E}_{\mathbb{K}}, \mathcal{F}_{\mathbb{K}}\right)
$$

the corresponding sheaves of holomorphic sections.

Lemma 6.3. The natural complex of sheaves

$$
0 \rightarrow\left(\mathcal{E}_{\mathbb{K}}, \mathcal{F}_{\mathbb{K}}\right) \rightarrow(\widetilde{\mathcal{E}}, \widetilde{\mathcal{F}}) \rightarrow(\mathcal{E}, \mathcal{F}) \rightarrow 0
$$

is exact.

Proof. We need to prove the sequence of stalks

$$
0 \rightarrow\left(\mathcal{E}_{\mathbb{K}}, \mathcal{F}_{\mathbb{K}}\right)_{z} \rightarrow(\widetilde{\mathcal{E}}, \widetilde{\mathcal{F}})_{z} \rightarrow(\mathcal{E}, \mathcal{F})_{z} \rightarrow 0
$$

is exact at each $z \in D^{2}$. When $z \in \operatorname{Int} D^{2}$, this immediately follows from the $\bar{\partial}$ Poincaré lemma. It remains to prove exactness when $z \in \partial D^{2}$. We will give details of the proof of surjectivity of the last map

$$
(\widetilde{\mathcal{E}}, \widetilde{\mathcal{F}})_{z} \rightarrow(\mathcal{E}, \mathcal{F})_{z}
$$

and leave the rest to the readers.

Let $z_{0} \in \partial D^{2}$. By choosing a sufficiently small neighborhood $U$ of $z_{0}$, we can holomorphically identify $\left.\left(E_{\mathbb{K}}, F_{\mathbb{K}}\right)\right|_{U}$ with the trivial bundle $\left(\mathbb{C}^{N-n}, \mathbb{R}^{N-n}\right) \rightarrow(U, U \cap$ $\left.\partial D^{2}\right)$. By shrinking $U$ if necessary, we may choose a holomorphic frame

$$
\left\{f_{1}, \cdots, f_{N-n}, f_{N-n+1}, \cdots, f_{N}\right\}
$$

of $(\widetilde{E}, \widetilde{F})$ so that $f_{j}=e_{j}, 1 \leq j \leq N-n$ the standard real constant basis of $\mathbb{R}^{N-n} \subset$ $\mathbb{C}^{N-n}$ and the projections of $\left\{\left[f_{N-n+1}\right], \cdots,\left[f_{N}\right]\right\}$ defines a holomorphic frame of $E$. 
Now let $\eta$ be a given holomorphic section of $E$ defined in a neighborhood $z \in$ $V \subset \bar{V} \subset U$ such that

$$
\left.\eta\right|_{V \cap \partial D^{2}} \in F
$$

We can write

$$
\eta=b_{N-n+1}\left[f_{N-n+1}\right]+\cdots+b_{N}\left[f_{N}\right]
$$

where $b_{j}$ 's are holomorphic functions on $V$. Then it is obvious that

$$
\xi_{\eta}:=b_{N-n+1} f_{N-n+1}+\cdots+b_{N} f_{N}
$$

defines a holomorphic section of $\widetilde{E}$ which projects to $\eta$. However $\xi_{\eta}$ may not satisfy the boundary condition

$$
\left.\left(\xi_{\eta}\right)\right|_{V \cap \partial D^{2}} \subset \widetilde{F}
$$

and so we need to correct it by adding a suitable holomorphic section of $\left(E_{\mathbb{K}}, F_{\mathbb{K}}\right) \cong$ $\left(\mathbb{C}^{N-n}, \mathbb{R}^{N-n}\right)$. Since $\left.\left(\xi_{\eta}\right)\right|_{\partial D^{2}} \subset F$, there exists a map $g: V \cap \partial D^{2} \rightarrow F=\mathbb{R}^{N-n}$, $g=\left(g_{1}, \cdots, g_{N-n}\right)$ such that

$$
\left.\xi_{\eta}\right|_{V \cap \partial D^{2}}(z)-\sum_{i=1}^{N-n} g_{i}(z) e_{i} \in \widetilde{F}
$$

for all $z \in V \cap \partial D^{2}$.

Now we solve the following Riemann-Hilbert problem for the map $G$ : $\left(D^{2}, \partial D^{2}\right) \rightarrow\left(\mathbb{C}^{N-n}, \mathbb{R}^{N-n}\right), G=\left(G_{1}, \cdots, G_{N-n}\right)$

$$
\left\{\begin{array}{l}
\frac{\partial G}{\partial \bar{z}}=0 \\
G(z)=g(z) \quad z \in V \cap \partial D^{2} .
\end{array}\right.
$$

It is well-known that this equation can be solved (see [O3] for example) on a neighborhood $V^{\prime} \subset \bar{V}^{\prime} \subset V$ by multiplying a cut-off function $\rho$ such that

$$
\rho(z)= \begin{cases}1 & \text { for } z \in \bar{V}^{\prime} \\ 0 & \text { for } z \quad \text { in a neighborhood of } \partial \bar{V} .\end{cases}
$$

Now it follows that if we define $\xi$

$$
\xi(z)=\xi_{\eta}(z)-\sum_{i=1}^{N-n} G_{i}(z) e_{i}
$$

it satisfies

$$
[\xi]=\left[\xi_{\eta}\right] \quad \text { and } \xi(z) \in \widetilde{F}_{z}, z \in V \cap \partial D^{2} .
$$

This finishes the proof of surjectivity of (6.3). $\mathrm{Q}$

The exact sequence (6.2) of the sheaves induces the long exact sequence of cohomology

$$
\begin{aligned}
0 & \rightarrow H^{0}\left(E_{\mathbb{K}}, F_{\mathbb{K}}\right) \rightarrow H^{0}(\widetilde{E}, \widetilde{F}) \rightarrow H^{0}(E, F) \longrightarrow \\
& \rightarrow H^{1}\left(E_{\mathbb{K}}, F_{\mathbb{K}}\right) \rightarrow H^{1}(\widetilde{E}, \widetilde{F}) \rightarrow H^{1}(E, F) \rightarrow 0 .
\end{aligned}
$$


Therefore to prove $H^{1}\left(D^{2}, \partial D^{2} ; E, F\right)=\{0\}$, it is enough to prove the following lemma.

Lemma 6.4. $H^{1}(\widetilde{E}, \widetilde{F})=\{0\}$.

Proof. From the definition of the bundle pair $(\widetilde{E}, \widetilde{F}) \rightarrow\left(D^{2}, \partial D^{2}\right)$, we have

$$
\widetilde{E}=D^{2} \times \mathbb{C}^{N}, \quad \widetilde{F}=\ell_{1} \oplus \cdots \oplus \ell_{N}
$$

Here for each $j=1, \cdots, N, \ell_{j}$ is the line bundle which is the tangent space of the circle

$$
\theta \mapsto e^{2 \pi \mu_{j} \theta} \cdot c_{j} \subset \mathbb{C}
$$

with $\mu_{j} \geq 0$ is an integer given in Theorem 5.2. Now the lemma immediately follows from the study of the one-dimensional Riemann-Hilbert problem with this Lagrangian loop (see e.g., [O3] for this kind of analysis).

This finishes the proof of the vanishing result

$$
H^{1}(E, F)=\{0\}
$$

and so the discs $w$ obtained in Theorem 5.2 and so all the discs in $X$ with boundary lying on $L$ are Fredholm-regular. $\square$

Now, let us consider the compactification of the moduli space of holomorphic discs.

Corollary 6.5. Singular strata of the compactified moduli space of holomorphic discs which consist of only disc components, have expected dimensions.

Proof. First note that from the classification theorem, all evaluation maps from the moduli space to the Lagrangian submanifold are submersions. This also may be seen from the torus action on the space of holomorphic discs. Hence, any singular stratum which consists of only disc components is given by transversal intersections of appropriate moduli spaces of holomorphic discs. Hence it has expected dimension.

When there is a singular stratum with sphere bubble, it may cause a trouble, since the standard complex structure is not always Fredholm regular for holomorphic spheres. Hence, the moduli spaces of holomorphic spheres may have dimensions different from expected dimensions. But since we only evaluate only at the boundary of the discs (not on spheres), with Fano condition, the evaluation image of such stratum is always of codimension of two or higher. Hence, it is plausible that these moduli spaces with evaluation maps define well-defined currents on $L$. We still believe that the moduli chains in the general Fano toric case are rectifiable. Since we have not been able to prove this statement in general, we make a following additional assumption on the toric Fano manifold for the computation of Floer cohomology.

Assumption 6.1. The toric Fano manifold $M$ is assumed to be convex. Namely we require that for any genus 0 stable map $f: \Sigma \rightarrow M, f^{*} T_{M}$ is generated by global sections.

In fact, the above assumption may be replaced by the following slightly less restrictive condition.

1. All holomorphic spheres with chern number less than $(n+2) / 2$ in toric Fano manifold are Fredholm regular, or 
2. The Chern number of the holomorphic spheres are at least $(n+2) / 2$, where $2 n=\operatorname{dim}(X)$.

In the first case, all singular strata of the moduli space of holomorphic discs (relevant in Floer cohomology) will have expected dimensions. The convex algebraic manifolds (tangent sheaf is generated by global sections) satisfy the first assumption, hence complex projective spaces and products of complex projective spaces satisfy our assumption. In the second case, note that the moduli spaces of holomorphic discs with Maslov index greater than $(n+1)$ does not contribute to the Bott-Morse Floer cohomology by the dimension counting. And with the second assumption, all the relevant moduli spaces can not have sphere bubbles due to the index restriction, hence Floer cohomology is well-defined.

7. Holomorphic discs of Maslov index two. We briefly recall the definition of the Bott-Morse Floer coboundary operator from [FOOO], while refering readers for the precise definition to $[\mathrm{FOOO}]$ or $[\mathrm{Cho} 2]$. : For $[P, f] \in C^{*}(L, \mathbb{Q})$ and non-zero $\beta \in \pi_{2}(M, L)$,

$$
\left\{\begin{array}{l}
\delta_{\beta}([P, f])=\left(\mathcal{M}_{2}(\beta)_{e v_{1}} \times{ }_{f} P, e v_{0}\right) \\
\delta_{0}([P, f])=(-1)^{n}[\partial P, f]
\end{array}\right.
$$

And the boundary operator is defined as

$$
\delta([P, f])=\sum_{\beta \in \pi_{2}(M, L)} \delta_{\beta}([P, f]) \otimes T^{\omega(\beta)} q^{\frac{\mu(\beta)}{2}} .
$$

And we extend it linearly over the universal Novikov ring $\Lambda_{\text {nov }}$. The following boundary property follows from the proof of [Theorem 6.24, FOOO] in which is considered the case where all the obstructions vanish, after combined with some additional cancellation arguments used in [addenda, O1], [Theorem 4.9, Cho] to deal with the case where the obstruction does not vanish but is a multiple of the fundamental cycle. We omit the proof referring to that of [Theorem 4.9, Cho].

Theorem 7.1. Assume that $X_{\Sigma(P)}$ is Fano and $L$ is as before. Then

$$
\delta \circ \delta=0 .
$$

Since the standard complex structure $J_{0}$ in these toric manifolds are regular as proved in the last section, we may proceed to compute the actual Floer boundary map with respect to $J_{0}$. The relevant calculations in our cases will be reduced to the study of discs of Maslov index two as in [Cho1] because of the following proposition.

Proposition 7.2. Let $\delta_{k}$ to be the formal sum of $\delta_{\beta}$ with $\mu(\beta)=k$. Then we have $\delta_{k} \equiv 0$ for $k \geq 4$.

Proof. Consider the homotopy class $\beta \in \pi_{2}(X, L)$ with the Maslov index $\mu(\beta) \geq 4$. The fiber product $\mathcal{M}_{2}(\beta)_{e v_{1}} \times_{f} P$ for the current Lagrangian torus fiber of the Fano toric manifolds is always transversal and so becomes a smooth manifold of dimension $\operatorname{dim}(P)+\mu(\beta)-1$. Now we consider the chain

$$
e v_{0}: \mathcal{M}_{2}(\beta)_{e v_{1}} \times_{f} P \rightarrow L .
$$

We would like to show that this is zero as a current on $L$. Note that if its image has dimension less than that of $\mathcal{M}_{2}(\beta)_{e v_{1}} \times_{f} P$, the value of the boundary operator of $P$ is zero as a current of dimension $\operatorname{dim}(P)+\mu(\beta)-1$ (See [FOOO] for details). 
It is immediate to check from the transversality of the fiber product that if all the elements in $\mathcal{M}(\beta)$ is of multiplicity greater than one, the dimension of the image of $e v_{0}$ is strictly less than that of $\mathcal{M}_{2}(\beta)_{e v_{1}} \times_{f} P(=\operatorname{dim}(P)+\mu(\beta)-1)$.

Therefore it remains to prove the statement in the case where generic elements in $\mathcal{M}(\beta)$ are simple. In this case, the lifting of $w$ in Theorem 5.2 must have the representation

$$
z_{j}(\widetilde{w})=c_{j} \prod_{k=1}^{\mu_{j}} \frac{z-\alpha_{j, k}}{1-\bar{\alpha}_{j, k} z}
$$

such that the set $\left\{\mu_{j}\right\}_{1 \leq k \leq N}$ of non-negative integers is relatively prime, i.e, does not have a common factor. In particular, there are at least two $\mu_{j}$ 's that are not zero since we assume $\mu(\beta) \geq 4$. For the simplicity of the exposition, we consider the case where $\mu_{1}=\mu_{2}=1$ and all others are zero. Other cases can be dealt similarly. In this case, we have $\mu(\beta)=4$ and so $\mathcal{M}_{2}(\beta)_{e v_{1}} \times_{f} P$ have dimension $\operatorname{dim} P+3$, unless it is empty.

Noting that

$$
e v_{0}\left(\mathcal{M}_{2}(\beta)_{e v_{1}} \times_{f} P\right)=\bigcup_{p \in P} e v_{0}\left(\mathcal{M}_{2}(\beta)_{e v_{1}} \times_{f}\langle p\rangle\right)
$$

it is enough to consider the case that $P$ is a point cycle $\langle p t\rangle$ in $X$. The fiber product $\mathcal{M}_{2}(\beta)_{e v_{1}} \times_{f}\langle p t\rangle$ has dimension 3 and the image $e v_{0}\left(\mathcal{M}_{2}(\beta)_{e v_{1}} \times_{f} P\right)$ is nothing but the set of the boundary image points of holomorphic discs in $\mathcal{M}(\beta)$ that meets the point $\langle p t\rangle$.

We claim that in the case of the current Lagrangian torus fiber of the Fano toric manifolds this latter image cannot have dimension bigger than two. To prove this, we consider the lifts of the holomorphic discs to $U(\Sigma)$. From the expression of lifted discs in Theorem 5.2 and by the uniqueness of the liftings upto the action of $T(\Sigma)$, the image of the boundary of the lifted discs is the union

$$
\bigcup_{\substack{t \in T(\Sigma), z \in \partial D^{2}}} t \cdot\left(\frac{z-\alpha_{1}}{1-\bar{\alpha}_{1} z}, \frac{z-\alpha_{2}}{1-\bar{\alpha}_{2} z}, 1 \cdots, 1\right) \subset \mathbb{C}^{N} .
$$

However it follows from Theorem 5.2 that the projection of this to $L$ by $\pi$, which is precisely the image $e v_{0}\left(\mathcal{M}_{2}(\beta)_{e v_{1}} \times_{f}\langle p t\rangle\right) \subset L$, has dimension always less than or equal to two. This finishes the proof for the case $\mu_{1}=\mu_{2}=1$. Other cases can be done similarly. $\mathrm{Q}$

Based on this theorem, we will be mainly interested in the holomorphic discs of Maslov index two for the computation of $H F^{B M}\left(L ; J_{0}\right)$. From the classification theorem, it is easy to see that there exists exactly $N$ distinct holomorphic discs of Maslov index 2 (up to an automorphism of the disc) passing a given point in $L$. We denote the homotopy class of such discs by $\beta_{j} \in \pi_{2}(X, L)$ for $j=1, \cdots, N$ :

Definition 7.1. For the homogeneous coordinates $z_{1}, \cdots, z_{N}$, we denote by $D\left(v_{j}\right)$ the holomorphic disc of class $\beta_{j} \in \pi_{2}(X, L)$ associated to the lifted disc

$$
\left\{\begin{array}{l}
z_{k}=c_{k} \text { for } k \neq j \\
z_{j}=c_{j} \cdot z
\end{array}\right.
$$

for $z \in D^{2}$, where $\left(c_{1}, \cdots, c_{N}\right) \in\left(\mathbb{C}^{*}\right)^{N}$ are chosen to satisfy the boundary condition. 
Now we want to express each such disc in terms of the coordinates of the torus $\left(\mathbb{C}^{*}\right)^{n} \subset X_{\Sigma(P)}$ to compute the boundary operator. Recall that in toric varieties, the torus $\left(\mathbb{C}^{*}\right)^{n}$ corresponds to 0 -cone in $N$ or the dual cone $M_{\mathbb{R}}$.

$$
\left(\mathbb{C}^{*}\right)^{n} \cong \operatorname{Spec} \mathbb{C}\left[x_{1}, x_{1}^{-1}, x_{2}, x_{2}^{-1}, \cdots, x_{n}, x_{n}^{-1}\right] .
$$

Its coordinate can also be obtained by applying Proposition 2.2 for the cone $\sigma$ which is generated by the standard basis vectors $\left\langle e_{1}, \cdots, e_{n}\right\rangle$ (Such cone may not exist in the fan $\Sigma$, but the coordinate expression of $\left(\mathbb{C}^{*}\right)^{n}$ obtained this way is still true).

Hence we use Proposition 2.2 to find the relation with the $\left(\mathbb{C}^{*}\right)^{n}$ coordinates and the homogeneous coordinates. If we choose the generators of the cone $\left(v_{i_{j}}\right)$ in Proposition 2.2 to be $\left\langle e_{1}, \cdots, e_{n}\right\rangle$, its dual basis becomes

$$
u_{i_{j}}=e_{j}^{*} .
$$

From the equation (2.3), we have

$$
\left\{\begin{array}{c}
x_{1}^{\sigma}=z_{1}^{\left\langle v_{1}, e_{1}^{*}\right\rangle} \cdots z_{N}^{\left\langle v_{N}, e_{1}^{*}\right\rangle} \\
\vdots \\
x_{n}^{\sigma}=z_{1}^{\left\langle v_{1}, e_{n}^{*}\right\rangle} \cdots z_{N}^{\left\langle v_{N}, e_{n}^{*}\right\rangle} .
\end{array}\right.
$$

Hence for the holomorphic disc $D\left(v_{j}\right)$, by substituting (7.3) into the above equations, we get the following :

$$
\left\{\begin{array}{c}
x_{1}^{\sigma}=c_{1}^{\prime} \cdot z^{\left\langle v_{j}, e_{1}^{*}\right\rangle}=c_{1}^{\prime} \cdot z^{v_{j}^{1}} \\
\vdots \\
x_{n}^{\sigma}=c_{n}^{\prime} \cdot z^{\left\langle v_{j}, e_{n}^{*}\right\rangle}=c_{n}^{\prime} \cdot z^{v_{j}^{n}}
\end{array}\right.
$$

where $v_{j}=\left(v_{j}^{1}, \cdots, v_{j}^{n}\right)$.

Proposition 7.3. For $i=1, \cdots, N$, the holomorphic disc $D\left(v_{j}\right)$ given by (7.3) can be written in terms of coordinates of the torus $\left(\mathbb{C}^{*}\right)^{n}$ as

$$
\left(C_{1} z^{v_{j}^{1}}, C_{2} z^{v_{j}^{2}}, \cdots, C_{n} z^{v_{j}^{n}}\right)
$$

where constants $C_{i} \in \mathbb{C}$ are chosen to satisfy the given Lagrangian boundary condition.

EXAmple 7.2. For the Clifford torus case, the holomorphic discs of index two are

$$
\left[z: c_{1}: \cdots: c_{n}\right], \cdots,\left[1: c_{1}: \cdots: c_{n} z\right],
$$

which in the standard open set $U_{0}$ are

$$
\left(c_{1} \frac{1}{z}, \cdots, c_{n} \frac{1}{z}\right),\left(c_{1} z, \cdots, c_{n}\right), \cdots,\left(c_{1}, \cdots, c_{n} z\right) .
$$

Now, the image of the moment map of $\mathbb{P}^{n}$ is the standard $n$ simplex, which can be written as follows:

$$
\begin{gathered}
\text { For } v_{1}=e_{1}, v_{n}=e_{n}, v_{n+1}=(-1,-1, \cdots,-1) \in \mathbb{R}^{n}, \\
\left\{\begin{array}{l}
\left\langle x, v_{i}\right\rangle \geq 0 \text { for } i \leq n \\
\left\langle x, v_{n+1}\right\rangle \geq-1 .
\end{array}\right.
\end{gathered}
$$


Now one can see the theorem is true in this case.

$$
\left\{\begin{array}{l}
v_{n+1} \Longrightarrow\left(c_{1} \frac{1}{z}, \cdots, c_{n} \frac{1}{z}\right) \\
v_{j} \Longrightarrow\left(c_{1}, \cdots, c_{j} z, \cdots, c_{n}\right) .
\end{array}\right.
$$

We have the classification theorem, Theorem 5.2 in terms of the homogeneous coordinates, but it is also convenient to look at them in the open sets $\mathbb{C}^{n}$ corresponding to $n$-dimensional cones in $\Sigma$. But one should note that not all discs are contained in these affine open sets. More precisely, if the holomorphic disc intersects with $V\left(v_{i_{1}}\right), \cdots, V\left(v_{i_{j}}\right)$ (possibly at different points), and if $\left\{v_{i_{1}}, \cdots, v_{i_{j}}\right\}$ is a primitive collection, then such disc can not be contained in the affine open sets. But as the primitive collections have two or more elements, the discs of Maslov index two which intersect only one of the submanifolds $V\left(v_{j}\right)$ 's are always contained in the affine open sets.

Proposition 7.4. For the affine open set $\mathbb{C}^{n}$ corresponding to $n$-dimensional cone $\sigma=\left\langle v_{i_{1}}, \cdots, v_{i_{n}}\right\rangle$ in $\Sigma$, the holomorphic discs with Maslov index 2 contained in this open set $\mathbb{C}^{n} \subset X$ are just $D\left(v_{i_{1}}\right), \cdots, D\left(v_{i_{n}}\right)$ up to an automorphism of a disc.

Proof. For such an open set $\mathbb{C}^{n} \subset X$, the Lagrangian torus fiber $L$ is defined by $\left|z_{i}\right|=c_{i}$ for $i=1, \cdots, n$ for some $c_{i} \in \mathbb{R}$. And the holomorphic discs which are mapped into this open set $\mathbb{C}^{n}$ are indeed easy to classify. More precisely, the $i$-th coordinate of such maps are just given by the Blaschke products times the constant $c_{i}$. Hence, holomorphic discs of Maslov index 2 are (up to automorphism of disc) can be written in terms of coordinates of $\mathbb{C}^{n}$ as

$$
\begin{gathered}
\left(c_{1} z, c_{2}, \cdots, c_{n}\right) \\
\left(c_{1}, c_{2} z, \cdots, c_{n}\right) \\
\vdots \\
\left(c_{1}, c_{2}, \cdots, c_{n} z\right) .
\end{gathered}
$$

As the coordinate of $\mathbb{C}^{n}$ is determined by the dual cone $\check{\sigma}$ of the cone $\sigma=$ $\left\langle v_{i_{1}}, \cdots, v_{i_{n}}\right\rangle$. The primitive generators of $\check{\sigma}$ are given by the dual $\mathbb{Z}$-basis $\left\langle u_{1}, \cdots, u_{n}\right\rangle$ in $M$ since $X$ is smooth.

Let $z_{1}, \cdots, z_{n}$ be the coordinates of the torus $\left(\mathbb{C}^{*}\right)^{n} \subset X$ given by $M_{\mathbb{R}}$. From [Ful], the affine coordinates $x_{1}^{\sigma}, \cdots, x_{n}^{\sigma}$ are given by the primitive generators as follows: For $u_{i}:=\left(u_{i 1}, \cdots, u_{i n}\right) \in M$,

$$
\begin{cases}x_{1}^{\sigma} & =z_{1}^{u_{11}} z_{2}^{u_{12}} \cdots z_{n}^{u_{1 n}} \\ & \vdots \\ x_{n}^{\sigma} & =z_{1}^{u_{n 1}} z_{2}^{u_{n 2}} \cdots z_{n}^{u_{n n}} .\end{cases}
$$

Then, the torus coordinates $z_{k}$ can be recovered from the affine coordinates $x_{1}^{\sigma}, \cdots, x_{n}^{\sigma}$ : Take

$$
\begin{aligned}
\left(x_{1}^{\sigma}\right)^{v_{i_{1}}^{k}} \cdot\left(x_{2}^{\sigma}\right)^{v_{i_{2}}^{k}} \cdots\left(x_{n}^{\sigma}\right)^{v_{i_{n}}^{k}} & =z_{1}^{\left(u_{11} v_{i_{1}}^{k}+\cdots+u_{n 1} v_{i_{n}}^{k}\right)} \cdots z_{n}^{\left(u_{1 n} v_{i_{1}}^{k}+\cdots+u_{n n} v_{i_{n}}^{k}\right)} \\
& =z_{1}^{\left(V^{t} \cdot U\right)_{1 k}} \cdots z_{n}^{\left(V^{t} \cdot U\right)_{n k}}=z_{k}
\end{aligned}
$$


where $U, V$ are $(n \times n)$ matrices whose $j$-th rows are given by the vectors $v_{i_{j}}, u_{j}$ respectively. The last equality follows from the duality between $v_{i_{j}}$ and $u_{j}$.

Hence the holomorphic disc in $\mathbb{C}^{N}$ given by

$$
\left(c_{1}, \cdots, c_{j} z, \cdots, c_{N}\right)
$$

can be rewritten in the coordinates of the torus $\left(\mathbb{C}^{*}\right)^{n}$ as

$$
\left\{\begin{aligned}
z_{1} & =\left(x_{1}^{\sigma}\right)^{v_{i_{1}}^{1}} \cdots\left(x_{n}^{\sigma}\right)^{v_{i_{n}}^{1}}=C_{1} \cdot z^{v_{i_{j}}^{1}} \\
& \vdots \\
z_{n} & =\left(x_{1}^{\sigma}\right)^{v_{i_{1}}^{n}} \cdots\left(x_{n}^{\sigma}\right)^{v_{i_{n}}^{n}}=C_{n} \cdot z^{v_{i_{j}}^{n}}
\end{aligned}\right.
$$

for $\left(C_{1}, \cdots, C_{n}\right) \in\left(\mathbb{C}^{*}\right)^{n}$. This is nothing but the expression of the disc $D\left(v_{i_{j}}\right)$ in Proposition 7.3. This proves the proposition.

8. The areas of holomorphic discs. In this section we compute the symplectic areas of the holomorphic discs. For each such holomorphic disc $D\left(v_{j}\right)$, there exists $S^{1}$-action on its image from the torus action on the toric variety. From the coordinate expression of holomorphic discs in Theorem 7.3, this $S^{1}$ can be easily seen as a subgroup of $T=\left(S^{1}\right)^{n}$ via the monomorphism

$$
S^{1} \rightarrow T: e^{i \theta} \mapsto\left(e^{i v_{j}^{1} \theta}, \cdots, e^{i v_{j}^{n} \theta}\right)
$$

for each given $j=1, \cdots, N$. We will fix one such $j$ in the rest of this section.

In the level of Lie algebra, the $S^{1} \subset T$ is generated by the element

$$
\xi=v_{j}^{1} e_{1}+v_{j}^{2} e_{2}+\cdots+v_{j}^{n} e_{n} \in \operatorname{Lie}\left(T^{n}\right) \cong \mathbb{R}^{n} .
$$

From now on, we denote by $\mu_{T}$ for the moment map of the whole torus $(T \cong$ $\left.\left(S^{1}\right)^{n}\right)$ action. The image $\mu_{T}\left(D\left(v_{j}\right)\right)$ of holomorphic discs $D\left(v_{j}\right)$ under the moment map $\mu_{T}$ can be easily seen to be 1-dimensional because it is invariant under the $S^{1}$ action generated by $\xi$, and it meets with the boundary of the moment polytope because when the disc meets the submanifold $V\left(v_{j}\right)$. The intersection point is a fixed point of the $S^{1}$ action we described above. Indeed, $\mu_{T}\left(D_{j}\right)$ meets the hyperplane defined by

$$
\left\langle x, v_{j}\right\rangle=\lambda_{j}
$$

since the preimage under the moment map $\mu_{T}$ of this hyperplane has the stabilizer $v_{j}$. Also recall that the image of the Lagrangian torus fiber under $\mu_{T}$ is a point, which we denote by

$$
A=\left(a_{1}, a_{2}, \cdots, a_{n}\right) \in\left(\mathbb{R}^{n}\right)^{*} .
$$

Let $(r, \theta)$ be the standard polar coordinate of $D^{2}(1) \subset \mathbb{C}$ and consider the map

$$
(r, \theta) \mapsto \mu_{T}(w(r, \theta))
$$

where

$$
w=D\left(v_{j}\right):\left(D^{2}, \partial D^{2}\right) \rightarrow(X, L)
$$


provided in Proposition 7.3. Since the disc is invariant under the $S^{1}$-action (8.1), the map is independent of $\theta$. We write the corresponding curve by

$$
\alpha:[0,1] \rightarrow\left(\mathbb{R}^{n}\right)^{*}=\left(\operatorname{Lie}\left(T^{n}\right)\right)^{*} ; \quad \alpha(r):=\mu_{T}(w(r, \cdot)) .
$$

We are now ready to prove the following area formula of the disc $D\left(v_{j}\right)$, which will play a crucial role later when we relate our computation of the Floer cohomology to Hori-Vafa's Landau-Ginzburg $B$-model calculation.

Theorem 8.1. The area of the holomorphic disc $D\left(v_{j}\right)$ in Proposition 7.3 is

$$
2 \pi\left(\left\langle A, v_{j}\right\rangle-\lambda_{j}\right) .
$$

Proof. Let $\eta \in \operatorname{Lie}\left(T^{n}\right)$ be any element and $\eta_{X}$ be the vector field on $X$ generated by $\eta$. By definition of the moment map $\mu_{T}$, we have the following defining formula of the moment map

$$
\left.d\left\langle\mu_{T}, \eta\right\rangle=\eta_{X}\right\rfloor \omega_{P}
$$

in general $[\mathrm{MW}]$. We apply this identity to $\eta=\xi$ defined in (8.2) to have

$$
\left.d\left\langle\mu_{T}, \xi\right\rangle=\xi_{X}\right\rfloor \omega_{P}
$$

Therefore we derive

$$
\begin{aligned}
\frac{d}{d r}\langle\alpha(r), \xi\rangle & =\left\langle d \mu_{T}\left(\frac{\partial w}{\partial r}\right), \xi\right\rangle \\
& =d\left\langle\mu_{T}, \xi\right\rangle\left(\frac{\partial w}{\partial r}\right) \\
& \left.=\xi_{X}\right\rfloor \omega_{P}\left(\frac{\partial w}{\partial r}\right)
\end{aligned}
$$

where we regard $\mu_{T}$ both as the map from $X$ to $\left(\operatorname{Lie}\left(T^{n}\right)\right)^{*}$ and as a $\left(\operatorname{Lie}\left(T^{n}\right)\right)^{*}$-valued function.

And it follows from the coordinate formula (7.6) that

$$
\xi_{X}(w(r, \theta))=\frac{\partial w}{\partial \theta}(r, \theta)
$$

By substituting this into (8.4), we have derived

$$
\frac{d}{d r}\langle\alpha(r), \xi\rangle=\omega_{P}\left(\frac{\partial w}{\partial \theta}, \frac{\partial w}{\partial r}\right) .
$$

From this, we derive

$$
\begin{aligned}
\operatorname{Area}\left(D\left(v_{j}\right)\right) & =\int_{D^{2}} w^{*} \omega_{P}=\int_{0}^{1} \int_{0}^{2 \pi} \omega_{P}\left(\frac{\partial w}{\partial r}, \frac{\partial w}{\partial \theta}\right) d \theta d r \\
& =-2 \pi \int_{0}^{1} \frac{d}{d r}\langle\alpha(r), \xi\rangle d r \\
& =2 \pi(\langle\alpha(0), \xi\rangle-\langle\alpha(1), \xi\rangle) .
\end{aligned}
$$

The value of $\alpha(1) \equiv \mu_{T}(w(1, \theta))$ is the base of the Lagrangian torus fiber $L$ which is nothing but $\langle A, \xi\rangle$ and $\alpha(1)$ is in the hyperplane determined by

$$
\langle x, \xi\rangle=\lambda_{j} .
$$

Therefore we have proved that the area of the disc is $2 \pi\left(\langle A, \xi\rangle-\lambda_{j}\right)$. Finally noting that $\xi=v_{j}$ in (8.2), we have finished the proof. $\square$ 
9. Standard spin structure. We recall the notion of the standard spin structure introduced in [Cho1] for the case of the Clifford torus in $\mathbb{P}^{n}$. A spin structure of $L$ is equivalent to the homotopy class of a trivialization of the tangent bundle of $L$ over the one skeleton of $L$, which can be extended over two skeleton. We also recall that a framing of the manifold $L$ is defined to be the homotopy class of a trivialization of the tangent bundle $T L$. Therefore each framing canonically fixes a spin structure of $L$.

Recall that a Lie group has a natural framing of its tangent bundle. In particular a Lie group with an orientation has a preferred spin structure. Now a torus $\left(S^{1}\right)^{n}=$ $\mathbb{R}^{n} / \mathbb{Z}^{n}$ has a canonical orientation on its Lie algebra which is canonically isomorphic to $\mathbb{R}^{n}$. And we have a canonical framing of $\left(S^{1}\right)^{n}$. Then, any orbit of the torus $\left(S^{1}\right)^{n}$ in toric manifolds has a canonically induced trivialization and orientation. We fix the orientation of a torus orbit to be the induced orientation. And we call the induced spin structure on any torus orbit standard spin structure.

For the space of holomorphic discs with a given homotopy class, say $\widetilde{\mathcal{M}}(\beta)$, consideration of the deformations of discs via torus action gives the subspace of the tangent space $T \widetilde{\mathcal{M}}(\beta)$. This subspace can be oriented by the orientation of the torus group, which determines the orientation of the moduli space of holomorphic discs via the Proposition 21.3 of [FOOO]. Also note that there exists $2^{n}=\left|H^{1}(L ; \mathbb{Z} / 2)\right|$ different spin structures for the torus $L$. Other spin structures besides the standard one can be naturally considered in the setting of the Floer cohomology twisted by the flat line bundles on $L$ : Calculations of the Floer cohomology with different spin structures can be substituted by the Floer cohomology twisted by the flat line bundles on $L$ with holonomy $e^{\pi i}$ along appropriate generators of $\pi_{1}(L)$.

Our computations will be carried out in the rest of the paper in terms of the standard spin structure. We refer readers to [Cho1] for more detailed discussions on the orientation and computations for different spin structures.

10. Computation of the Bott-Morse Floer cohomology. Now, we are ready to compute the Bott-Morse Floer cohomology of any Lagrangian torus fiber $L$ in symplectic toric manifold $X_{\Sigma(P)}$. We will assume in this section that $X_{\Sigma(P)}$ is Fano.

The Bott-Morse Floer cohomology defined in section 7 satisfies

$$
\delta \circ \delta=0
$$

for our torus fiber. Note that we do not need to deform the boundary operator of the Floer complex by introducing obstruction cycles since all non-constant holomorphic discs have positive Maslov indices in our case.

We fix the standard spin structure of $L$, which fixes the orientation of the moduli space of holomorphic discs. The orientation of the boundary (7.1) not only depends on the orientation of the moduli space $\mathcal{M}_{2}(\beta)$, but also the fiber product orientation. It was studied in great detail in [Cho1], [FOOO], and so we restrict our discussion about orientation to a minimum.

Recall that the Floer cochain complex in [FOOO] is constructed using currents. From now on, the cycles we write actually represents their Poincaré duals, and we will not distinguish homology $H_{*}(L, \mathbb{Q})$ and cohomology $H^{*}(L, \mathbb{Q})$ in our presentation.

The filtration on the boundary operator $\delta$ with energy induces a spectral sequence $E_{r}^{*, *}$ which converges to the Floer cohomology $H F^{B M}\left(L ; J_{0}\right)$. Recall from [FOOO] that

$$
E_{2}^{p, q} \cong\left(H^{*}(L, \mathbb{Q}) \otimes e^{q}\right)^{p}
$$


where ()$^{p}$ means the total degree $p$. To compute the Floer cohomology, we work with this spectral sequence and the main step is to compute the boundary $\delta_{2}$ of the cohomology generators. Here $\delta_{2}$ is the boundary operator given by considering only Maslov index 2 discs.

We first compute the boundary for a point class $\langle p t\rangle$. We denote the generators of $H_{*}(L, \mathbb{Q})$ by $L_{1}, \cdots, L_{n}$. More precisely, by $L_{j}$ we denote a cycle given by the image of the map

$$
S^{1} \rightarrow\left(\mathbb{C}^{*}\right)^{n}: e^{i \theta} \mapsto\left(c_{1}, \cdots, c_{j} e^{i \theta}, \cdots, c_{n}\right) .
$$

In view of Proposition 7.3 and considering an orientation as in [Cho1], we have

$$
\delta_{\beta_{j}}\langle p t\rangle=(-1)^{n}\left(v_{j}^{1} L_{1}+\cdots+v_{j}^{n} L_{n}\right)
$$

where $\beta_{j}=\left[D\left(v_{j}\right)\right] \in \pi_{2}(X, L)$. Hence,

$$
\begin{aligned}
\delta_{2}(\langle p t\rangle) & =\sum_{j=1}^{N}(-1)^{n} T^{\operatorname{Area}\left(\beta_{j}\right)} \cdot q \cdot\left(v_{j}^{1} L_{1}+\cdots+v_{j}^{n} L_{n}\right) \\
& =\sum_{j=1}^{N}(-1)^{n} T^{2 \pi\left(\left\langle v_{j}, A\right\rangle-\lambda_{j}\right)} \cdot q \cdot\left(v_{j}^{1} L_{1}+\cdots+v_{j}^{n} L_{n}\right) .
\end{aligned}
$$

We can also compute the Floer cohomology with flat line bundle $\mathcal{L}$ on it, which we denote by

$$
H F^{B M}\left((L, \mathcal{L}) ; J_{0}\right)
$$

If we denote by

$$
h_{\alpha}=e^{i \nu_{\alpha}}
$$

the holonomy of the line bundle $\mathcal{L}$ along the cycle $L_{\alpha}$ for $\alpha=1, \cdots, n$, Proposition 5.2 implies that the holonomy along the boundary of the disc $D\left(v_{j}\right)$, becomes

$$
h_{1}^{v_{j}^{1}} \cdots h_{n}^{v_{j}^{n}}=e^{i\left\langle\nu, v_{j}\right\rangle}:=h^{v_{j}}
$$

where the vector $\nu=\nu_{\mathcal{L}}$ is defined by

$$
\nu=\left(\nu_{1}, \cdots, \nu_{n}\right)
$$

which we call the holonomy vector of $\mathcal{L}$.

In this case, the boundary operator of the Floer cochain complex is defined as follows [Fu2]:

$$
\left\{\begin{array}{l}
\delta_{\beta}([P, f])=\left(\mathcal{M}_{2}(\beta)_{e v_{1}} \times_{f} P, e v_{0}\right) \cdot\left(h l_{\partial \beta} \mathcal{L}\right) \otimes q \text { for } \beta \neq 0 \\
\delta_{0}([P, f])=(-1)^{n}[\partial P, f] .
\end{array}\right.
$$

Therefore, we have

$$
\delta_{2}(\langle p t\rangle)=\sum_{j}(-1)^{n} h^{v_{j}} T^{2 \pi\left(\left\langle v_{j}, A\right\rangle-\lambda_{j}\right)} \cdot q \cdot\left(v_{j}^{1} L_{1}+\cdots+v_{j}^{n} L_{n}\right) .
$$


By identifying $H_{1}(L: \mathbb{Q})$ with $\mathbb{Q}^{n}$ via $L_{i} \mapsto e_{i}$, we may write the condition to have $\delta_{2}(\langle p t\rangle)=0$ as

$$
\sum_{j}(-1)^{n} h^{v_{j}} T^{2 \pi\left(\left\langle v_{j}, A\right\rangle-\lambda_{j}\right)} \cdot v_{j}=0 .
$$

It is not hard to see that if $\delta_{2}(\langle p t\rangle)=0$, we would have $\delta_{2}(P)=0$ in $H^{*}(L, \mathbb{Q})$ for any cycle $P \in H_{*}(L, \mathbb{Q})$ (see [Cho] for the relevant computations). Therefore, in this case, the Floer cohomology $\operatorname{HF}^{B M}\left((L, \mathcal{L}) ; J_{0}\right)$ is isomorphic to the singular cohomology of $L$. In particular, it is non-vanishing. The following proposition implies that, one only needs to consider $\delta_{2}(\langle p t\rangle)$ for the computation of Floer cohomology.

TheOREM 10.1. If $\delta_{2}(\langle p t\rangle)=0$, then Bott-Morse Floer cohomology is isomorphic to the singular cohomology of $L$ as a $\Lambda_{\text {nov }}$-module, i.e.,

$$
H F^{B M}\left((L, \mathcal{L}) ; J_{0}\right) \cong H^{*}\left(L ; \Lambda_{\text {nov }}^{\mathbb{C}}\right)
$$

where $\Lambda_{\text {nov }}^{\mathbb{C}}$ is the Novikov ring twisted by the line bundle $\mathcal{L}$ in an obvious way.

If $\delta_{2}(\langle p t\rangle) \neq 0$, then the Floer cohomology $\operatorname{HF}^{B M}\left((L, \mathcal{L}) ; J_{0}\right)$ vanishes.

Proof. It remains to prove the second statement. Suppose $\delta_{2}(\langle p t\rangle) \neq 0$, and consider the lowest energy terms of $\delta_{2}\langle p t\rangle$ which gives rise to a non-zero term: Suppose the terms with this energy are given by $\delta_{\beta_{i_{1}}}, \cdots, \delta_{\beta_{i_{\ell}}}$. Denote by $\widetilde{\delta}_{2}$ the sum

$$
\widetilde{\delta}_{2}:=\delta_{\beta_{i_{1}}}+\cdots+\delta_{\beta_{i_{\ell}}} .
$$

By the assumption $\delta_{2}\langle p t\rangle \neq 0$, we have $\widetilde{\delta}_{2} \neq 0$. It follows from the construction of the spectral sequence in [FOOO] that this becomes the boundary operator of the spectral sequence of a certain step, say $r$. From our choice of $\beta_{i_{*}}$, lower energy terms give rise to zero boundary maps in the spectral sequence. Therefore we have,

$$
E_{r}^{p, q} \cong E_{2}^{p, q} \cong\left(H^{*}(L, \mathbb{Q}) \otimes e^{q}\right)^{p} .
$$

We will show that

$$
E_{r+1} \cong 0
$$

For this we will compute $\widetilde{\delta}_{2}$ for the cohomology generators of $H^{*}(L, \mathbb{Q})$. $T^{\text {Area }} q$,

In $H^{*}(L, \mathbb{Q})$, we may write, omitting the common factor of formal parameter

$$
\widetilde{\delta}_{2}\langle p t\rangle=c_{1}\left[L_{1}\right]+c_{2}\left[L_{2}\right]+\cdots+c_{n}\left[L_{n}\right] .
$$

At least one of $c_{i}$ is non-zero from our assumption. It is not hard to see that

$$
\widetilde{\delta}_{2}\left\langle L_{i}\right\rangle=\sum_{j=1}^{n} c_{j}\left\langle L_{j} \times L_{i}\right\rangle
$$

where $L_{i} \times L_{i}$ is 0 -cycle. Or more generally,

$$
\widetilde{\delta}_{2}\left(L_{i_{i}} \times L_{i_{2}} \times \cdots \times L_{i_{k}}\right)=\sum_{j=1}^{n} c_{j}\left\langle L_{j} \times\left(L_{i_{i}} \times L_{i_{2}} \times \cdots \times L_{i_{k}}\right)\right\rangle
$$


where the latter is a 0 -cycle if $j \in\left\{i_{1}, i_{2}, \cdots, i_{k}\right\}$ (See [Cho1] for the case of Clifford torus in $\mathbb{P}^{n}$ ).

From now on, for index sets, say $J$ with $j=|J|$ elements, we denote its elements as $J=\left\{j_{1}, \cdots, j_{j}\right\}$ with $j_{1}<j_{2}<\cdots<j_{j}$. And we denote $J_{\widehat{s}}=J \backslash\left\{j_{s}\right\}$.

Now we denote an arbitrary element of $k$ dimensional cycles as

$$
\sum_{I,|I|=k} A_{I} L_{I}
$$

for $I \subset\{1,2, \cdots, n\}$ and $A_{I} \in \mathbb{Q}$. The boundary of this element is

$$
\begin{aligned}
\widetilde{\delta}_{2}\left(\sum_{I,|I|=k} A_{I} L_{I}\right) & =\sum_{I} A_{I}\left(\widetilde{\delta}_{2} L_{I}\right) \\
& =\sum_{I} A_{I}\left(c_{1} L_{1}+\cdots+c_{n} L_{n}\right) \times L_{I} \\
& =\sum_{J,|J|=k+1} \sum_{s=1}^{k+1} A_{J_{\widetilde{s}}}(-1)^{s-1} c_{j_{s}} L_{J} .
\end{aligned}
$$

Hence, the element $\sum_{I,|I|=k} A_{I} L_{I}$ is in the kernel of $\widetilde{\delta}_{2}$ if for any set $J \subset$ $\{1,2, \cdots, n\}$ with $|J|=k+1$, the following equation holds:

$$
\sum_{s=1}^{k+1} A_{J_{\widehat{s}}}(-1)^{s-1} c_{j_{s}}=0 .
$$

Set

$$
\left\{\begin{array}{l}
S:=\left\{i \in\{1,2, \cdots, n\} \mid c_{i}=0\right\} \\
S^{c}:=\{1,2, \cdots, n\} \backslash S .
\end{array}\right.
$$

Then, the equation (10.8) is exactly the same equation as we had in Theorem 13.3 in [Cho1] with $\left(h_{i_{s}}-h_{0}\right)$ replaced by $c_{i_{s}}$.

Hence, by applying the same method, one can show that such elements in the kernel of $\widetilde{\delta}_{2}$ lies in the image of $\widetilde{\delta}_{2}$. This finishes the proof. $\square$

Since for a fiber to has a non-trivial Floer cohomology is a very special geometric property, it seems to deserves a name to them.

DEFINITION 10.1. We call balanced a Lagrangian fiber that has a non-vanishing Floer cohomology.

In the next section, we will provide a geometric description of balanced torus fibers.

11. Description of the balanced torus fibers. In this section, we now examine the equation (10.6) in terms of toric geometry. In particular, in the case of no line bundle twisted, we provide a concrete toric description of the conditions for a fiber to satisfy the equation.

For given $A \in$ intP, we partition $G=G(\Sigma)=\left\{v_{j}\right\}_{1 \leq j \leq N}$ into the disjoint union

$$
G=\coprod_{a} G_{(A ; a)}
$$


where $G_{(A ; a)}$ is the set of $v_{j} \in G$ with the symplectic area of the associated homotopy class $\beta_{j}=\left[D\left(v_{j}\right)\right] \in \pi_{2}(X, L)$

$$
\omega_{P}\left(\beta_{j}\right)=a
$$

for each given positive number $a$. Obviously $G_{(A ; a)}=\emptyset$ except for a finite number of values of $a$ 's

$$
0<a_{1}<a_{2}<\cdots<a_{L_{A}}
$$

and $1 \leq L_{A} \leq N$. Then (10.6) becomes

$$
\sum_{v_{j} \in G_{\left(A ; a_{\ell}\right)}} h^{v_{j}} v_{j}=\sum_{v_{j} \in G_{\left(A ; a_{\ell}\right)}} e^{i\left\langle\nu, v_{j}\right\rangle} v_{j}=0
$$

for all $1 \leq \ell \leq L_{A}$.

Proposition 11.1. Assume $X_{\Sigma(P)}$ is Fano and let $L=\mu^{-1}(A) \subset\left(X_{\Sigma(P)}, \omega_{P}\right)$ be a fiber for $A \in$ int $P$ and $\mathcal{L}$ be a flat line bundle with the holonomy vector $\nu=$ $\left(\nu_{1}, \cdots, \nu_{n}\right)$ such that $A$ and $\nu$ satisfy (11.1). Then we have the isomorphism

$$
H F^{B M}\left((L, \mathcal{L}) ; J_{0}\right) \cong H^{*}\left(L ; \Lambda_{\text {nov }}^{\mathbb{C}}\right) .
$$

For all other cases, $\operatorname{HF}^{B M}\left((L, \mathcal{L}) ; J_{0}\right)$ is trivial.

Now we specialize to the case without $\mathcal{L}$, i.e., all $h^{v_{j}} \equiv 1$. In the remaining section, we will provide a more concrete description of the balanced fibers by analyzing (11.1) in terms of toric data.

Note that in this case (11.1) just becomes

$$
\sum_{v_{j} \in G_{\left(A ; a_{\ell}\right)}} v_{j}=0
$$

We denote by

$$
\{1, \cdots, N\}=\coprod_{\ell=1}^{L_{A}} I_{\ell}
$$

the partition of $\{1, \cdots, N\}$ corresponding to the partition of $G=\coprod_{\ell=1}^{L_{A}} G_{\left(A ; a_{\ell}\right)}$. We also denote

$$
e_{I_{\ell}}=\sum_{j \in I_{\ell}} e_{j}
$$

where $\left\{e_{j}\right\}$ are the standard basis vectors of $\mathbb{Z}^{n}$. By the exact sequence

$$
0 \rightarrow \mathbb{K} \stackrel{i}{\rightarrow} \mathbb{Z}^{N} \stackrel{\pi}{\rightarrow} \mathbb{Z}^{n} \rightarrow 0
$$

(11.2) implies that there exists $\delta_{\ell} \in \mathbb{K}$ such that

$$
i\left(\delta_{\ell}\right)=e_{I_{\ell}} \in \mathbb{Z}^{I_{\ell}}
$$

for each $1 \leq \ell \leq L_{A}$, where $\mathbb{Z}^{I_{\ell}}$ is the obvious product space. We denote by

$$
\Delta_{\ell} \subset\left(S^{1}\right)^{I_{\ell}}
$$


the obvious diagonal circle group generated by the vector $e_{I_{\ell}} \in \mathbb{Z}^{I_{\ell}}$ and by $\Delta$ their products as a subgroup of $\left(S^{1}\right)^{N}$. By construction, we have

$$
\Delta \subset K
$$

with $\operatorname{dim} \Delta=L_{A} \leq \operatorname{dim} K=N-n$.

We will now carry out the "reduction by stages" to describe our toric manifolds $X_{\Sigma(P)}$ and the Lagrangian torus fiber $L$ in a two-step process. We denote $\delta=\operatorname{Lie}(\Delta)$ and by

$$
\mu_{\Delta}: \mathbb{C}^{N} \rightarrow \delta^{*}
$$

the moment map of the action of $\Delta$ on $\mathbb{C}^{N}$. We denote

$$
j: \Delta \hookrightarrow K\left(\subset\left(S^{1}\right)^{N}\right) \quad \text { or } j: \delta \hookrightarrow k\left(\subset \mathbb{R}^{N}\right)
$$

the inclusion homomorphism and $T^{\Delta}:=\left(S^{1}\right)^{N} / \Delta$.

We note that $\left(S^{1}\right)^{N}$ acts on $\mathbb{C}^{N}$ as the direct product of the actions of $d_{\ell^{-}}$ dimensional torus $\left(S^{1}\right)^{I_{\ell}}$ on $\mathbb{C}^{I_{\ell}}$. By carrying out the first reduction by the action of $\Delta$, we have obtained the reduced space

$$
\begin{aligned}
& Y_{\Delta}=\mu_{\Delta}^{-1}\left(j^{*}(r)\right) / \Delta \cong \mathbb{P}^{\left(d_{1}-1\right)} \times \cdots \times \mathbb{P}^{\left(d_{L_{A}}-1\right)} \\
& \omega_{\Delta}=\omega_{1} \oplus \cdots \oplus \omega_{L_{A}}
\end{aligned}
$$

where we have

$$
j^{*}(r)=\sum_{\ell=1}^{L_{A}}\left(-\lambda_{I_{\ell}}\right) e_{I_{\ell}}^{*} \in \delta^{*}, \quad \lambda_{I_{\ell}}=\sum_{i \in I_{\ell}}\left(\lambda_{i}\right)
$$

with respect to the basis $\left\{e_{I_{1}}^{*}, \cdots, e_{I_{\ell}}^{*}\right\}$ dual to the basis $\left\{e_{I_{1}}, \cdots, e_{I_{\ell}}\right\}$ of $\delta$, and $\omega_{\ell}$ is the Fubini-Study form on $\mathbb{P}^{\left(d_{\ell}-1\right)}$ associated to the value $\lambda_{I_{\ell}}$ of the momentum function $\mu_{\Delta_{\ell}}: \mathbb{C}^{d_{\ell}} \rightarrow \delta_{\ell}^{*} \cong \mathbb{R}$, which becomes nothing but the standard momentum function of the $S^{1}$ action on $\mathbb{C}^{d_{\ell}}$ i.e.,

$$
z \in \mathbb{C}^{d_{\ell}} \mapsto \frac{1}{2}|z|^{2} \in \mathbb{R}
$$

Furthermore the residual torus $T^{\Delta}=\left(S^{1}\right)^{N} / \Delta$ is the direct product

$$
T^{\Delta}=\prod_{\ell} T^{\Delta_{\ell}}
$$

with $T^{\Delta_{\ell}}:=\left(S^{1}\right)^{I_{\ell}} / \Delta_{\ell}$, and canonically acts on the reduced space $Y_{\Delta}$ as the direct product action of the standard torus action of $T^{\Delta_{\ell}} \cong\left(S^{1}\right)^{d_{\ell}} / \Delta_{\ell}$ on $\mathbb{P}^{\left(d_{\ell}-1\right)}$. We denote

$$
t^{\Delta_{\ell}}=\operatorname{Lie}\left(\left(S^{1}\right)^{I_{\ell}} / \Delta_{\ell}\right) \cong \mathbb{R}^{\left(d_{\ell}-1\right)} .
$$

This action of the torus $T^{\Delta_{\ell}}$ on $\mathbb{P}^{\left(d_{\ell}-1\right)}$ naturally extends to the action of the product

$$
U^{\Delta}:=\prod_{\ell} U\left(d_{\ell}\right)
$$


as the Kähler isometry with respect to the canonical complex and symplectic structures induced from the ones on $Y_{\Delta}=\mu_{\Delta}^{-1}\left(j^{*}(r)\right) / \Delta$.

Now the quotient group $K / \Delta:=K_{\Delta}$ acts on $Y_{\Delta}$. We denote its moment map by

$$
\mu_{K_{\Delta}}: Y_{\Delta} \rightarrow k_{\Delta}^{*}
$$

and the natural projection $K \rightarrow K_{\Delta}$ by $\pi_{\Delta}$. Then we have the identity

$$
\pi_{\Delta}^{*} \circ \mu_{K_{\Delta}}=\mu_{K}
$$

and the second reduction provides the description of $\left(X_{\Sigma(P)}, \omega_{P}\right)$ as the reduced space

$$
X_{\Sigma(P)} \cong \mu_{K_{\Delta}}^{-1}(s) / K_{\Delta}
$$

where $s \in k_{\Delta}^{*}$ such that $\pi_{\Delta}^{*}(s)=r$.

In terms of this identification, the Lagrangian torus $L=\mu^{-1}(A), A=\left(a_{1}, \cdots, a_{n}\right)$ can be written as

$$
\mu_{T^{\Delta}}^{-1}\left(A^{1}, \cdots, A^{L_{A}}\right) / K_{\Delta} \cong\left(\mu_{T^{\Delta_{1}}}^{-1}\left(A^{1}\right) \times \cdots \times \mu_{T^{\Delta_{L_{A}}}}^{-1}\left(A^{L_{A}}\right)\right) / K_{\Delta}
$$

where $A^{\ell} \in\left(t^{\Delta_{\ell}}\right)^{*}$ and $\left(A^{1}, \cdots, A^{L_{A}}\right) \in \oplus_{\ell}\left(t^{\Delta_{\ell}}\right)^{*}$ and

$$
\mu_{T^{\Delta_{\ell}}}: \mathbb{P}^{\left(d_{\ell}-1\right)} \rightarrow\left(t^{\Delta_{\ell}}\right)^{*} \cong \mathbb{R}^{\left(d_{\ell}-1\right)}
$$

is the standard moment map on $\mathbb{P}^{\left(d_{\ell}-1\right)}$ of the action by the torus $T^{\Delta_{\ell}}$. Here $\left(A^{1}, \cdots, A^{L_{A}}\right)=\pi^{*}(A)$ where

$$
\pi: T^{\Delta}=\left(S^{1}\right)^{N} / \Delta \rightarrow \frac{\left(S^{1}\right)^{N} / \Delta}{K / \Delta} \cong\left(S^{1}\right)^{N} / K=T^{n} .
$$

By the symmetry consideration, it follows that $\mu_{T^{\Delta_{\ell}}}^{-1}\left(A^{\ell}\right)$ is the Clifford torus of $\mathbb{P}^{\left(d_{\ell}-1\right)}$.

We summarize the above discussion into the following theorem

Theorem 11.2. Let $X_{\Sigma(P)}=\mu^{-1}(r) / K$ be a Fano toric manifold with the symplectic form $\omega_{P}$ associated to the polytope $P$. Then each balanced Lagrangian torus fiber in $X_{\Sigma(P)}$ has the form

$$
L \cong\left(L_{1} \times \cdots \times L_{L_{A}}\right) / K_{\Delta} \subset \mu_{K_{\Delta}}^{-1}(s) / K_{\Delta} \cong X_{\Sigma(P)}
$$

where $K_{\Delta}=K / \Delta$ and $L_{\ell}$ is the Clifford torus of $\left(\mathbb{P}^{\left(d_{\ell}-1\right)}, \omega_{\ell}\right)$ with $\omega_{\ell}$ the Fubini-Study form associated to the normalization

$$
\mathbb{P}^{d_{\ell}-1}=\mu_{\Delta_{\ell}}^{-1}\left(-\lambda_{I_{\ell}}\right) / S^{1}, \quad \lambda_{I_{\ell}}=\sum_{i \in I_{\ell}} \lambda_{i}
$$

12. Hori-Vafa's B-Model Calculation. In this section and the next, we will relate the equation (10.6) with the critical point equation of the superpotential of the Landau-Ginzburg mirror to the toric manifold $\left(X_{\Sigma(P)}, \omega_{P}\right)$, after substituting $T^{2 \pi}=e^{-1}$. We will closely follow the notations from [HV] with few minor exceptions, and exclusively use convention that the letter $i$ runs over $1, \cdots, N, a$ over $1, \cdots, k=$ $N-n)$ and $\alpha$ over $1, \cdots, n$. 
In this section, we first describe the prediction of Floer cohomology by Hori via the mirror symmetry correspondence from Hori and Vafa $[\mathrm{HV}]$ or Hori $[\mathrm{H}]$.

Suppose the $k$-dimensional torus $K=\left(S^{1}\right)^{k}$ acts on $\mathbb{C}^{N}$ as follows.

$$
\left(e^{i \theta_{1}}, \cdots, e^{i \theta_{k}}\right) \cdot\left(z_{1}, \cdots, z_{N}\right)=\left(\sum_{a=1}^{k} e^{i Q_{1 a} \theta_{a}} z_{1}, \cdots, \sum_{a=1}^{k} e^{i Q_{N a} \theta_{a}} z_{N}\right) .
$$

The moment map of this action is given by

$$
\begin{gathered}
\mu: \mathbb{C}^{N} \rightarrow\left(\mathbb{R}^{k}\right)^{*} \\
\left(z_{1}, \cdots, z_{N}\right) \mapsto \frac{1}{2}\left(\sum_{i} Q_{i 1}\left|z_{i}\right|^{2}, \cdots, \sum_{i} Q_{i k}\left|z_{i}\right|^{2}\right)
\end{gathered}
$$

(See section 3 for more detailed discussion on this). Now we consider the quotient $\mu^{-1}(r) / K$ as toric manifolds where $r=\left(r_{1}, \cdots, r_{k}\right)$ lies in $\left(\mathbb{R}^{k}\right)^{*}$.

With some physical arguments, Hori and Vafa $[\mathrm{HV}]$ introduce the dual geometry by introducing periodic variables $Y_{i}, i=1, \cdots, N$ with $Y_{i} \equiv Y_{i}+2 \pi i$ such that for $a=1, \cdots, k$,

$$
\sum_{i=1}^{N} Q_{i a} Y_{i}=t_{a}
$$

where $t_{a}=r_{a}-i \theta_{a}$.

REMARK 12.1. Here we consider the case where the B-field is zero.

The real part of $Y_{i}$ represents the position of the Lagrangian torus fiber and imaginary part represents the holonomy of the line bundle on this torus fiber. And one considers the superpotential

$$
W:=\sum_{i=1}^{N} e^{-Y_{i}} .
$$

The critical points of the superpotential correspond to specific fibers and holonomies whose Floer cohomology are non-vanishing.

For a given $Q$, we consider the equation

$$
\sum_{i=1}^{N} v_{i} Q_{i a}=0, \quad v_{i} \in \mathbb{Z}
$$

The space of solutions of (12.3) form an integral lattice of rank $n=N-k$ in $\mathbb{R}^{N}$. We denote a $\mathbb{Z}$-basis of this lattice by $\left\{v^{\alpha}\right\}_{1 \leq \alpha \leq n} \subset \mathbb{R}^{N}$ with

$$
v^{\alpha}=\left(v_{1}^{\alpha}, \cdots, v_{N}^{\alpha}\right)
$$

each of them satisfying

$$
\sum_{i=1}^{N} v_{i}^{\alpha} Q_{i a}=0
$$


Therefore the general solutions for the constraint equation $\sum_{i} Q_{i a} Y_{i}=t_{a}$ have the form

$$
Y_{i}=\sum_{\alpha=1}^{n} v_{i}^{\alpha} \Theta_{\alpha}+y_{i}
$$

with $n=N-k$ periodic variables $\Theta_{\alpha}(\bmod 2 \pi i)$ where $y=\left(y_{1}, \cdots, y_{N}\right)$ is a special solution of

$$
\sum_{i=1}^{N} Q_{i a} Y_{i}=t_{a}
$$

(In [HV], the letters $t_{i}$ 's are used for $y_{i}$ 's which is somewhat confusing with the other usage of $t_{a}$ 's.)

Now the superpotential (12.2) of the mirror theory can be expressed as

$$
W=\sum_{i=1}^{N} \exp \left(-y_{i}-\left\langle\Theta, v_{i}\right\rangle\right),
$$

where

$$
v_{i}:=\left(v_{i}^{1}, v_{i}^{2}, \cdots, v_{i}^{n}\right) \in \mathbb{R}^{n} \cong N_{\mathbb{R}}
$$

and $\left\langle\Theta, v_{i}\right\rangle$ is the short hand notation for $\sum_{\alpha=1}^{n} v_{i}^{\alpha} \Theta_{\alpha}$. Note that the condition $\frac{\partial W}{\partial \Theta_{\alpha}}=0$ is the same as

$$
\sum_{i=1}^{N} e^{-Y_{i}} \cdot v_{i}^{\alpha}=\sum_{i=1}^{N} \exp \left(-y_{i}-\left\langle\Theta, v_{i}\right\rangle\right) \cdot v_{i}^{\alpha}=0
$$

for $\alpha=1, \cdots, n$. One can already see the similarity between equation (10.6) and the equation (12.7). In the next section, we show that two equations indeed coincide, if we substitute

$$
T^{2 \pi}=e^{-1}, \quad \text { and then } \quad y_{i}=-\lambda_{i} .
$$

13. Equivalence when $T^{2 \pi}=e^{-1}$. In this section, we show that our calculation of the (Bott-Morse) Floer cohomology indeed verifies the mirror symmetry prediction made by Hori-Vafa's $B$-model calculation. More precisely, the condition (10.6) to have non-vanishing Floer cohomology with $T^{2 \pi}=e^{-1}$ exactly corresponds to the critical points of the superpotential, with a canonical definition of the variables $Y_{i}$ 's.

To see the correspondence for the compact toric manifold $X_{\Sigma(P)}$, we define $Y_{i}$ as follows:

Definition 13.1. For $i=1, \cdots, N$, define $Y_{i} \in \mathbb{R} \times i(\mathbb{R} / 2 \pi \mathbb{Z})$ as

$$
\left\{\begin{array}{l}
\operatorname{Re}\left(Y_{i}\right)=\operatorname{Area}\left(\beta_{i}\right) / 2 \pi \\
\operatorname{Im}\left(Y_{i}\right)=i \log \left(h^{v_{i}}\right)=-\left\langle\nu, v_{i}\right\rangle \bmod 2 \pi
\end{array}\right.
$$

where $v_{i}$ 's are the generators of the one dimensional cones of the fan $\Sigma$ associated the toric manifold $X_{\Sigma(P)}$ as in section 2 and $\beta_{i} \in \pi_{2}(X, L)$ is its associated homology class, and $\nu$ is the holonomy vector of the flat line bundle $\mathcal{L}$ defined in (10.3). 
Then it follows from Theorem 8.1 that

$$
Y_{i}=\left(\left\langle A, v_{i}\right\rangle-\lambda_{i}\right)-i\left\langle\nu, v_{i}\right\rangle=\left\langle A-i \nu, v_{i}\right\rangle-\lambda_{i}
$$

and hence

$$
\begin{aligned}
e^{-Y_{i}} & =e^{-\left(\left\langle A-i \nu, v_{i}\right\rangle-\lambda_{i}\right)} \quad \text { or } \\
& =h^{v_{i}} e^{-\left(\left\langle A, v_{i}\right\rangle-\lambda_{i}\right)} .
\end{aligned}
$$

Consider the choice of $t_{a}$ 's given by the real numbers

$$
t_{a}=-\sum_{i=1}^{N} Q_{i a} \lambda_{i}
$$

for $a=1, \cdots, N-n$. Then by the choice of $t_{a}$ 's,

$$
y_{i}=-\lambda_{i}, \quad i=1, \cdots, N
$$

is a special solution of (12.1).

Proposition 13.1. For any vectors $A$ and $\nu$ above, $Y_{i}$ 's defined by (13.2) satisfy the constraint equation

$$
\sum_{i=1}^{N} Q_{i a} Y_{i}=t_{a} \text { for each } a=1, \cdots, N-n
$$

Proof. First note that we have the following equality from the exact sequence (2.1) or equation (12.4)

$$
\sum_{i=1}^{N} Q_{i a} v_{i}^{\alpha}=0 \quad \text { for all } \alpha
$$

and so we have

$$
\sum_{i=1}^{N} Q_{i a} v_{i}=0
$$

From this, we derive

$$
\begin{aligned}
\sum_{i=1}^{N} Q_{i a} Y_{i} & =\sum_{i=1}^{N} Q_{i a}\left(\left\langle A-i \nu, v_{i}\right\rangle-\lambda_{i}\right) \\
& =\left\langle A-i \nu, \sum_{i=1}^{N} Q_{i a} v_{i}\right\rangle-\sum_{i=1}^{N} Q_{i a} \lambda_{i} \\
& =0+t_{a}=t_{a}
\end{aligned}
$$

which finishes the proof. $\square$

Now identifying the variable $\Theta$

$$
\Theta=A-i \nu
$$


$Y_{i}$ 's defined in (13.2) coincide with (12.5).

Now, it remains to show that the condition (10.6) to have non-vanishing Floer cohomology corresponds to the critical points of the superpotential $W=\sum_{i=1}^{N} e^{-Y_{i}}$, if we substitute $T^{2 \pi}=e^{-1}$.

Proposition 13.2. The $\Theta=A-i \nu$ is a critical point of the superpotential $W$ if and only if $A$ and $\nu$ (or $h^{v_{i}}$ 's) satisfy (10.6), i.e., $\delta_{2}\langle p t\rangle=0$.

Proof. The condition (10.6)

$$
\sum_{i} h^{v_{i}} T^{2 \pi\left(\left\langle A, v_{i}\right\rangle-\lambda_{i}\right)} \cdot v_{i}=0
$$

becomes the following equation, after we substitute $T^{2 \pi}=e^{-1}$ :

$$
\sum_{i} h^{v_{i}} e^{-\left(\left\langle A, v_{i}\right\rangle-\lambda_{i}\right)} \cdot v_{i}=0
$$

Then from (13.4) and from the choice $y_{j}=-\lambda_{j}$, the above equation is same as

$$
\sum_{i} e^{-Y_{i}} v_{i}^{\alpha}=0 \forall \alpha
$$

which is precisely the condition for $Y_{i}$ to be the critical points of the superpotential $W$ as in the equation (12.7).

\section{Examples.}

14.1. The complex projective space $\mathbb{P}^{2}$. In [Cho], Floer cohomology of the Clifford torus $T^{2}$ with a flat line bundle is computed. This example extends the results by showing that there are no other fibers in $\mathbb{P}^{2}$ with non-vanishing Floer cohomology. We consider $\mathbb{P}^{2}$ associated with the moment polytope $P$ defined by

$$
\left\{\begin{array}{l}
\langle x,(1,0)\rangle \geq 0 \\
\langle x,(0,1)\rangle \geq 0 \\
\langle x,(-1,-1)\rangle \geq r .
\end{array}\right.
$$

Let $\left(a_{1}, a_{2}\right) \in \operatorname{int}(P)$. For the Lagrangian submanifold $L:=\mu_{T}^{-1}\left(a_{1}, a_{2}\right)$, there exist three Maslov index 2 discs (up to $\operatorname{Aut}\left(D^{2}\right)$ ) with boundary in $L$. It is not hard to check that its moment map $\mu_{T}$ trajectories are in fact straight lines. To find the torus fiber whose Floer cohomology is non-vanishing, we check the condition (10.6).

$$
\delta_{2}(\langle p t\rangle)=h_{1} T^{2 \pi a_{1}}(1,0)+h_{2} T^{2 \pi a_{2}}(0,1)+h_{1}^{-1} h_{2}^{-1} T^{2 \pi\left(-a_{1}-a_{2}+r\right)}(-1,-1)=0 .
$$

Since $h_{i} \in U(1)$, we have

$$
\begin{gathered}
\left\{\begin{array}{l}
h_{1}=h_{1}^{-1} h_{2}^{-2} \\
a_{1}=-a_{1}-a_{2}+r
\end{array}\right. \\
\left\{\begin{array}{l}
h_{2}=h_{1}^{-1} h_{2}^{-2} \\
a_{2}=-a_{1}-a_{2}+r .
\end{array}\right.
\end{gathered}
$$


Hence, we have

$$
\begin{gathered}
a_{1}=a_{2}=r / 3, \\
h_{1}=h_{2} \text { and } h_{1}^{3}=1 .
\end{gathered}
$$

The Lagrangian fiber $\mu_{T}^{-1}(r / 3, r / 3)$ is called the the Clifford torus and the holonomies $\left(h_{1}, h_{2}\right)$ of the line bundle $\mathcal{L}$ for the non-vanishing Floer cohomologies on the Clifford torus are

$$
(1,1),\left(e^{2 \pi i / 3}, e^{2 \pi i / 3}\right),\left(e^{4 \pi i / 3}, e^{4 \pi i / 3}\right) .
$$

14.2. Hirzebruch surface $F_{1}$. This example illustrates well the difference between the actual Floer cohomology with $\Lambda_{n o v}$-coefficient and the Floer cohomology with the parameter value $T^{2 \pi}=e^{-1}$. The latter was predicted by Hori-Vafa [HV] by the B-model calculation. However the latter version of the Floer cohomology is not invariant under the Hamiltonian isotopy of the Lagrangian torus fiber while the former version is so. The latter Floer cohomology has Euler number of fibers whose Floer cohomology is non-vanishing for toric Fano manifolds ([HV]), especially there exists four such fibers for Hirzebruch surface $F_{1}$. But we will show that the former version has no fiber whose Floer cohomology is non-vanishing.

We consider $F_{1}$ associated with the moment polytope $P_{1}$ defined by

$$
\left\{\begin{array}{l}
\langle x,(1,0)\rangle \geq-1 \\
\langle x,(0,1)\rangle \geq-1 \\
\langle x,(0,-1)\rangle \geq-1 \\
\langle x,(-1,1)\rangle \geq-1
\end{array}\right.
$$

(The compuation here can be generalized to the case of other polytopes). This polytope is reflexive [B2], thus $F_{1}$ is Fano. Let $\left(a_{1}, a_{2}\right) \in \operatorname{int}\left(P_{1}\right)$. For the Lagrangian submanifold $L:=\mu_{T}^{-1}\left(a_{1}, a_{2}\right)$, there exist four Maslov index 2 discs (up to $\operatorname{Aut}\left(D^{2}\right)$ ) with boundary in $L$. In the torus coordinates, these are given as

$$
\left(c_{1}, c_{2} z\right),\left(c_{1} z, c_{2}\right),\left(c_{1}, \frac{c_{2}}{z}\right),\left(\frac{c_{1}}{z}, c_{2} z\right)
$$

To find the torus fiber whose Floer cohomology is non-vanishing, we check the condition (10.6).

$$
\begin{array}{r}
0=\delta_{2}(\langle p t\rangle)=h_{1} T^{2 \pi\left(a_{1}+1\right)}(1,0)+h_{2} T^{2 \pi\left(a_{2}+1\right)}(0,1)+ \\
h_{2}^{-1} T^{2 \pi\left(-a_{2}+1\right)}(0,-1)+h_{1}^{-1} h_{2} T^{2 \pi\left(-a_{1}+a_{2}+1\right)}(-1,1) .
\end{array}
$$

From the first coordinate, we obtain,

$$
\left\{\begin{array}{l}
h_{1}=h_{1}^{-1} h_{2} \\
T^{2 \pi\left(a_{1}+1\right)}=T^{2 \pi\left(-a_{1}+a_{2}+1\right)} .
\end{array}\right.
$$

Therefore we have

$$
h_{2}=h_{1}^{2}, a_{2}=2 a_{1}
$$


From the second coordinate, we have

$$
h_{1}^{2} T^{2 \pi\left(2 a_{1}+1\right)}-h_{1}^{-2} T^{2 \pi\left(-2 a_{1}+1\right)}+h_{1} T^{2 \pi\left(a_{1}+1\right)}=0 .
$$

Or, equivalently

$$
\left(h_{1} T^{2 \pi a_{1}}\right)^{4}-1+\left(h_{1} T^{2 \pi a_{1}}\right)^{3}=0 .
$$

Now, we substitute $T^{2 \pi}=e^{-1}$. Then, the equation (14.7) becomes

$$
\left(h_{1} e^{-a_{1}}\right)^{4}-1+\left(h_{1} e^{-a_{1}}\right)^{3}=0 .
$$

By setting $X:=h_{1} e^{-a_{1}}$, we have

$$
X^{4}+X^{3}-1=0 .
$$

It is not hard to check the four solutions of this equation indeed gives the location of the four fibers inside the polytope $P_{1}$, whose Floer cohomology with the value $T^{2 \pi}=e^{-1}$ is non-vanishing. This agrees with the $\mathrm{B}$-model calculation from [HV].

For the Floer cohomology with $\Lambda_{0, n o v}$-coefficients, note that we regard $T$ as a formal parameter. Hence to have a solution of the equation (14.7), we should have

$$
a_{1}=0 .
$$

In this cases, the equation becomes,

$$
h_{1}^{4}+h_{1}^{3}-1=0 .
$$

It is easy to check that this equation does not have a solution for $h_{1} \in U(1)$. Hence, there exists no torus fiber in $F_{1}$ (from the polytope $P_{1}$ ) whose Floer cohomology with $\Lambda_{n o v}$-coefficient is non-vanishing.

Also note that the Hirzbruch surface $F_{1}$ is not convex, however, it satisfies the generalized assumption. Or one may observe that holomorphic discs of index 4 does not come into play in the computation of Floer cohomology due to dimensional reason, and it is clear that holomorphic discs of index two cannot bubble off the holomorphic sphere of chern number 1, since such holomorphic sphere does not intersect fiber tori whose moment map image lie in the interior of the moment polytope. Hence, Floer cohomology in this case is well-defined.

REMARK 14.1. For $F_{0} \cong \mathbb{P}^{1} \times \mathbb{P}^{1}$, it is easy to find the torus fiber (at the centor of the rectangle) with four possible holonomies whose Floer cohomology with $\Lambda_{n o v}$-coefficient is non-vanishing.

15. Obstruction classes, open Gromov-Witten invariants and superpotentials. Fukaya-Oh-Ohta-Ono [FOOO] have defined the obstruction cycles of the filtered $A_{\infty}$-algebra associated to each Lagrangian submanifold and developed a deformation theory thereof, which tells whether one can kill the $\mathfrak{m}_{0}$-term by a suitable gauge equivalence. The $\mathfrak{m}_{0}$ is defined by a collection of currents induced by the (co)chains

$$
\left[\mathcal{M}_{1}(\beta), e v_{0}\right]
$$

for all $\beta \in \pi_{2}(M, L)$. More precisely, we have

$$
\mathfrak{m}_{0}(1)=\sum_{\beta \in \pi_{2}(M, L)}\left[\mathcal{M}_{1}(\beta), e v_{0}\right] \cdot T^{\operatorname{Area}(\beta)} q^{\mu(\beta) / 2} \in C^{*}(L) \otimes \Lambda_{\text {nov }, 0} .
$$


The sequence $\left\{o_{k}(L)\right\}_{1 \leq k<\infty}$ of the obstruction classes introduced in [FOOO] is the iterative obstructions to deforming the filtered $A_{\infty}$-structure so that

$$
\mathfrak{m}_{0} \equiv 0 \quad \bmod T^{\lambda_{k+1}} \quad \text { as } k \rightarrow \infty .
$$

Here we order those $\lambda$ 's that appear as the area of $\beta$, i.e., as $\omega(\beta)$.

Since the paper [FOOO] appeared, it became a folklore among some mathematicians and physicists alike that under the mirror symmetry correspondence FOOO's obstruction (co)chain of the $A$-model should correspond to the Landau-Ginzburg superpotential of the $B$-model.

In fact, our computation confirms this test in the toric case. We now explain this correspondence precisely. We first recall from Theorem 5.2 and the orientability of the torus that there is no holomorphic discs of Maslov index less than 2. According to [section 7, FOOO], all obstruction classes $o(\beta)$ are well-defined and the only nontrivial obstruction classes (as currents) are the ones given by

$$
o(\beta):=\left[\mathcal{M}_{1}(\beta), e v_{0}\right] \quad \text { for } \beta \text { with } \mu(\beta)=2
$$

for the torus fibers in this paper (see [section 7, FOOO] for more explanation), which also coincides with $\mathfrak{m}_{0}(1)$ in this case. In view of Proposition 7.3 and consideration of the sign from [Cho], we also have

$$
o(\beta)=[L](=1)
$$

the fundamental class of $L$ for any $\beta$ with $\mu(\beta)=2$. Therefore we have obtained the formula for the obstruction class of $L$

$$
o(L)=\sum_{i=1}^{N} h^{v_{j}} T^{\operatorname{Area}\left(\beta_{j}\right)} \cdot q
$$

from the definition of obstruction classes [Definition $4.6 \& 4.8$, FOOO]. However it follows, by the same substitution $T^{2 \pi}$ by $e^{-1}$ as before, that the right hand side of (15.3) precisely becomes

$$
\sum_{i=1}^{N} \exp \left(-y_{i}-\left\langle\Theta, v_{i}\right\rangle\right)=W(\Theta)
$$

if we ignore the harmless grading parameter $q$. Therefore we have confirmed the exact correspondence

$$
o(L) \longleftrightarrow W
$$

for the case of Lagrangian torus fibers in toric manifolds.

In addition, comparing (10.6) with the derivative

$$
\frac{\partial W}{\partial \Theta}=\left(\frac{\partial W}{\partial \Theta_{1}}, \cdots, \frac{\partial W}{\partial \Theta_{n}}\right)
$$

after substitution of $T^{2 \pi}=e^{-1}$, we have also verified the correspondence

$$
\delta_{2}\langle p t\rangle \longleftrightarrow \frac{\partial W}{\partial \Theta}
$$


Recall from [addenda, O1] that the senior author observed that the obstruction class is determined by the (genus zero) one-point open Gromov-Witten invariants in the monotone case, which obviously generalizes to the toric Fano case (because the fiber does not have holomorphic discs of non-positive Maslov indices). Similarly in general, $\delta_{2}\langle p t\rangle$ is determined by the two-point open Gromov-Witten invariants from the definitions (7.1) and (7.2) [FOOO]. Here we would like to emphasize that in this case of torus fibers in the Fano toric manifolds, the (genus zero) open Gromov-Witten invariants considered here are rigorously well-defined (with respect to the canonical complex structure). This correspondence between adding one marked point and taking the derivative is consistent with the well-known principle in the calculus of correlation functions in physics. In the subsequent work, the first author showed the exact correspondence between products of $A_{\infty}$ algebra of Lagrangian submanifold and derivatives of the superpotential([Cho2]).

Combination of these facts suggests an intriguing relation between the derivatives of $W$ of the superpotential (or of the obstruction $o(L)$ ) and the "open Gromov-Witten invariants" of $L$ in general. (Here we put the quotation mark because the open Gromov-Witten invariants in general has not been rigorously defined.) In fact, there has been conjectured by physicists that the superpotential is related by the mirror symmetry correspondence to the "open Gromov-Witten potential" (see [KKLM] for example), and our work provides a concrete mathematical evidence via an $A$-model calculation. As far as we understand, most calculations, if not all, in the physics literature in this respect have been done in the $B$-model side. We hope to further investigate this relation in the future.

16. Discussion: non-convex cases. We believe that our calculation of the Floer cohomology in this paper should remain to be true for the non-Fano toric manifolds. In this section, we explain what remains to be proved for the non-Fano cases.

The structure and regularity theorem of smooth holomorphic discs still hold for the non-Fano case. However for singular curves, distinction occurs in the transversality problem because of the presence of multiple covered spheres of negative Chern numbers. Therefore it is essential to use the abstract perturbation in the framework of Kuranishi structure [FOn], even if all disc components are already regular. With this transversality problem taken care of, all the theorems, especially those in sections 4 and 10 should remain to be true, possibly except the statement

$$
H F\left(L, \phi(L) ; J^{\prime}\right) \cong H F^{B M}\left(L, J_{0}\right) .
$$

The proof of this isomorphism is expected to use a singular degeneration argument as those used in [FOh1] in the presence of non-trivial instantons which may not be transversal. One really has to construct a Kuranishi structure in the limit configurations and to prove other non-trivial convergence statements. This singular degeneration problem is currently being studied by the senior author with K. Fukaya [FOh2].

In the end, we expect that the above isomorphism still holds but details of the proof remain to be worked out. Because of this, we restrict ourselves to the Fano case for sections 4, 10 and the beginning of section 7 in this paper. 


\section{REFERENCES}

[Ar] ARnol'D, V. I., On a characteristic class entering the quantization conditions, Funct. Anal. Appl., 1 (1967), pp. 1-14.

[Au $\quad$ M. Audin, The Topology of Torus Actions on Symplectic Manifolds, Birkhäuser Verlag, Basel, Progress in Mathematics 93, 1991.

[B1] V. BATYREv, Quamtum cohomology rings of toric manifolds, Journees de geometrie Algebrique d'Orsay, 1992, Asterisque 218, pp. 9-34.

[B2] V. BATYRev, Dual polyhedra and mirror symmetry for Calabi-Yau hypersurfaces in toric varieties, J. Algebraic. Geom., 3:3 (1994), pp. 493-535.

[BC] P. Biran And K. CielebaK, Symplectic topology on subcritical manifolds, Comment. Math. Helv., 76:4 (2001), pp. 712-753.

[Che] Y. CheKanov, Lagrangian intersections, symplectic energy and areas of holomorphic curves, Duke Math. J., 95 (1998), pp. 213-226.

[Cho1] C.-H. Cho, Holomorphic discs, spin structures and the Floer cohomology of the Clifford torus, IMRN, 35 (2004), pp. 1803-1843.

[Cho2] C.-H. CHO, Products of Floer cohomology of Lagrangian torus fibers in toric Fano manifolds, Commun. Math. Phy., 260 (2005), pp. 613-640.

[Fl] A. Floer, Morse theory for Lagrangian intersections, J. Differ. Geom., 28 (1988), pp. 513547.

[Fu1] K. Fukaya, Morse homotopy, $A_{\infty}$-category and Floer homologies, in Proceedings of GARC Workshop on Geometry and Topology, ed by H. J. Kim, Seoul National University, Korea 1933.

[Fu2] K. FukAYA, Floer homology and mirror symmetry, Winter School on Mirror Symmetry, Vector Bundles and Lagrangian Submanifolds, Cambridge, MA, 1999, pp. 15-43, AMS/IP Stud. Adv. Math., 23, Amer. Math. Soc., Providence, RI, 2001.

[FOh1] K. FukaYA And Y.-G. OH, Zero-loop open strings in the cotangent bundle and Morse homotopy, Asian J. Math., 1 (1997), pp. 99-180.

[FOh2] K. FukAYA AND Y.-G. OH, in preparation.

[FOOO] K. Fukaya, Y.-G. OH, H. OHta AND K. Ono, Lagrangian intersection Floer theoryanomaly and obstruction, Kyoto University preprint, 2000; revision 2006.

[FOn] K. Fukaya And K. Ono, Arnold conjecture and Gromov-Witten invariants, Topology, 38 (1999), pp. 933-1048.

[Ful] W. Fulton, Introduction to Toric Varieties, Annals of Mathematics Studies, 131, Princeton University Press, Princeton, 1993.

[Gu] V. Guillemin, Kähler structures on toric varieties, J. Differ. Geom., 43 (1994), pp. 285309.

[H] K. HoRI, Linear models in supersymmetric D-branes, Proceedings of the KIAS conference-Mirror Symmetry and Symplectic Geometry- (Seoul, 2000), eds by K. Fukaya, Y.-G. Oh, K. Ono and G. Tian, World Sci. Publishing, River Edge, New Jersey, 2001.

[HV] K. Hori ANd C. VAFA, Mirror symmetry, preprint, 2000, hep-th/0002222.

[KKLM] S. Kachru, S. Katz, A. Lawrence and J. MCGreevy, Open string instantons and superpotentials, Phys. Rev. D62, 026001 (2000).

[KL] S. KATZ AND C.C. LIU, Enumerative geometry of stable maps with Lagrangian boundary conditions and multiple covers of the disc, Adv. Theor. Math. Phys., 5 (2001), pp. 149.

[Ko] M. Kontsevich, Homological algebra of mirror symmetry, ICM-1994 Proceedings, Zürich, Birkhäuser, 1995.

[LM] H. B. Lawson, Jr and M. L. Michelsohn, Spin Geometry, Princeton Mathematical Series, 38, 1986.

[MW] J. Marsden And A. Weinstein, Reduction of symplectic manifolds with symmetry, Reports on Math. Phys., 5 (1974), pp. 121-130.

[O1] Y.-G. OH, Floer cohomology of Lagrangian intersections and pseudo-holomorphic discs I, Comm. Pure and Appl. Math., 46 (1993), pp. 949-994 addenda, ibid, 48 (1995), pp. 1299-1302.

[O2] Y.-G. OH, Floer cohomology of Lagrangian intersections and pseudo-holomorphic discs II \& III, Comm. Pure Appl. Math. 46 (1993), pp. 995-1012 \& The Floer Memorial Volume (ed. by H. Hofer et al.), Birkhäuser, Basel, pp. 555-573, 1995.

[O3] Y.-G. OH, Riemann Hilbert problem and application to the perturbation theory of analytic discs, Kyungbook Math. J., 35:1 (1995), pp. 39-75.

[O4] Y.-G. OH, Floer cohomology, spectral sequence, and the Maslov class of Lagrangian em- 
beddings, IMRN, 7 (1996), pp. 305-346.

[O5] Y.-G. OH, On the structure of pseudo-holomorphic discs with totally real boundary conditions, J. Geom. Anal., 7:2 (1997), pp. 305-327.

[O6] Y.-G. OH, Spectral invariants, analysis of the Floer moduli space, and geometry of the Hamiltonian diffeomorphism group, Duke Math. J., 130 (2005), pp. 199-295.

[P] L. Polterovich, Hofer's diameter and Lagrangian intersections, Internat. Math. Res. Notices, 4 (1998), pp. 217-223.

[Se] P. SeIDEL, Lagrangian two-spheres can be symplectically knotted, J. Differ. Geom., 52:1 (1999), pp. 145-171.

[TY] R. ThOmas And S.T. YAU, Special Lagrangians, stable bundles and mean curvature flow, Comm. Anal. Geom., 10:5 (2002), pp. 1075-1113.

[Wi] E. Witten, Phases of $N=2$ theories in two dimensions, Nucl. Phys., B403 (1993), pp. 159-222. 